\title{
VdThit, a Thiamine Transport Protein, Is Required for Pathogenicity of the Vascular Pathogen Verticillium dahliae
}

\author{
Xiliang Qi, ${ }^{1,2}$ Xiaofeng Su, ${ }^{1}$ Huiming Guo, ${ }^{1}$ Juncang Qi, ${ }^{2}$ and Hongmei Cheng ${ }^{1}$ \\ ${ }^{1}$ Biotechnology Research Institute, Chinese Academy of Agricultural Sciences, Beijing 100081, China; and ${ }^{2}$ Agriculture College \\ of Shihezi University, Shihezi 832000, China
}

Accepted 7 April 2016.

\begin{abstract}
Verticillium dahliae causes a serious wilt disease of important crops and is difficult to control. Few plasma-membrane transport proteins for nutrient acquisition have been identified for this fungus, and their involvement in the disease process is unknown. Here, a plasma-membrane protein, the $V$. dahliae thiamine transporter protein VdThit, was characterized functionally by deletion of the VdThit gene in $V$. dahliae. Disruption strains were viable, but growth and conidial germination and production were reduced and virulence was impaired. Interestingly, by supplementing exogenous thiamine, growth, conidiation, and virulence of the VdsThit mutants were partially restored. Stress-tolerance assays showed that the VdDThit mutant strains were markedly more susceptible to oxidative stress and UV damage. Highpressure liquid chromatography-mass spectrometry (HPLCMS) and gas chromatography-mass spectrometry (GC-MS) analyses showed low levels of pyruvate metabolism intermediates acetoin and acetyl coenzyme A (acetyl-CoA) in the VdsThit mutant strains, suggesting that pyruvate metabolism was suppressed. Expression analysis of VdThit confirmed the importance of VdThit in vegetative growth, reproduction, and invasive hyphal growth. Furthermore, a green fluorescent protein (GFP)-labeled $V d \Delta T h i t$ mutant (VdDThit-7-GFP) was suppressed in initial infection and root colonization, as viewed with light microscopy. Together, these results showed that VdThit plays an indispensable role in the pathogenicity of V. dahliae.
\end{abstract}

Verticillium dahliae is a soil-borne, filamentous fungal pathogen that causes a vascular wilt in annual and perennial herbaceous plants and woody angiosperms (Hillocks 1992; Klosterman et al. 2009) and several billions of dollars of annual crop losses throughout the world (Cai et al. 2009; Fradin and Thomma 2006; Klosterman et al. 2009). As such, V. dahliae has been likened to a bottleneck in commercial crop productivity; its microsclerotia can survive for many years in soil and dead plant tissues, making the disease difficult to control with pesticides (Cai et al. 2009; Klosterman et al. 2011). Gaining insight into the signaling pathways and molecular machinery responsible for pathogenicity of the fungus should inform new strategies to

Corresponding author: H. Cheng; Telephone and Fax +86 10 82106125; E-mail: chenghongmei@caas.cn

*The $\boldsymbol{e}$-Xtra logo stands for "electronic extra" and indicates that two supplementary figures are published online.

(c) 2016 The American Phytopathological Society prevent infection by $V$. dahliae (Fradin and Thomma 2006; Klosterman et al. 2011). However, only a few studies have focused on pathogenicity-related molecular mechanisms in the fungus and the identification or functional characterization of resistance-related molecular mechanisms in the host (Luo et al. 2014). Thus, characterization of fungal development and pathogenicity-related molecular mechanisms is urgently needed for developing strategies to control the fungus.

The long-lived microsclerotia of $V$. dahliae are induced to germinate in the soil by plant root exudates and hyphae then colonize the root surface (Pegg and Brady 2002). Hyphae penetrate the epidermal cell layer without forming appressoria and grow intracellularly and intercellularly through the root cortex, entering the xylem vessels, changing the endogenous environment of the plant cells, and forming a parasitic environment (Klosterman et al. 2009; Pegg and Brady 2002; Reusche et al. 2012; Zhao et al. 2014). In this parasitic environment, the hyphae take up plant nutrients to colonize the host tissue further and eventually conidiate (Fradin and Thomma 2006). The conidia are transported within the xylem vessels in stems and leaves through the plant by its transpiration flow (Eynck et al. 2007). When the hyphae colonize the plant's entire vascular system, flow in small areas can be disrupted, resulting in the wilt phenotype-plant stunting and wilting, vascular browning, and foliar chlorosis and necrosis (Pegg and Brady 2002). Finally, microsclerotia form in the parenchyma of the dying stem and are released into the soil to complete the life cycle (Klosterman et al. 2009).

Our knowledge of the mechanism of pathogenesis of $V$. dahliae, however, has remained at a preliminary exploratory stage. Secreted pathogenic proteins are known to play important roles during the initial infection process, which includes degradation of the host cell walls, scavenging of reactive oxygen species (ROS) generated by the host, stress responses, and protein metabolism (Chu et al. 2015; Hogenhout et al. 2009). How nutrients from the host plant are absorbed and ultimately enhance virulence when $V$. dahliae colonizes host plants is still unknown. Therefore, exploring V. dahliae pathogenesis and finding the key protein factors involved in fungal metabolism during invasion and colonization are very important.

The soluble B vitamin (B1) thiamine is essential for all organisms as a key cofactor of enzymes with an important role in carbohydrate metabolism (Butterworth 2003; Rapala-Kozik 2011). Only bacteria, fungi, and plants can naturally synthesize thiamine; animals and humans must obtain their thiamine from food (Friedrich 1987). For fungi, thiamine is also essential for vegetative growth, development, sporulation, host invasion, and adaptation to environmental stress (Hohmann and Meacock 1998; Rapala-Kozik 2011). 
The thiamine molecule consists of a thiazole and a pyrimidine moiety, which are synthesized independently at different branches of the thiamine biosynthetic pathway (Hohmann and Meacock 1998; Young 1986). After their independent formation, 2-methyl-4-amino-5-hydroxymethylpyrimidine pyrophosphate (HMP-PP) and 4-methyl-5-(2-hydroxy-ethyl) thiazole monophosphate (TH-P) are condensed to form thiamine monophosphate, which is finally pyrophosphorylated to the active thiamine pyrophosphate (TPP) (Mojzita and Hohmann 2006). Fungal organisms either take up thiamine from the medium or synthesize it de novo, but they primarily acquire exogenous thiamine, using a thiamine transporter protein in their plasma membrane (Mojzita and Hohmann 2006). In Saccharomyces cerevisiae, the thiamine transporter protein is Thi7p (Thi10) (Enjo et al. 1997; Singleton 1997).

The thiamine transporter protein is a high-affinity transporter for thiamine, belonging to the reduced folate carrier transporter family, and transports extracellular thiamine into the cell (Dutta et al. 1999; Lagarde et al. 2004; Larkin et al. 2012). Targeted deletion of the SLC19a2 gene (encoding a high-affinity thiamine transporter) in mice on a thiamine-free diet led to diabetes, sensorineural deafness, and megaloblastosis (Oishi et al. 2002). However, the thiamine transporter protein itself has not yet been reported in $V$. dahliae and other fungi, except for $S$. cerevisiae. Additionally, how a limited nutrient supply is taken up by plasma-membrane transporter proteins and how it contributes to pathogenicity are poorly understood in this filamentous fungus. In an effort to better understand $V$. dahliae virulence and pathogenesis, a $V$. dahliae $\Delta$ Thit deletion mutant strain was generated. The growth and development, its infection and colonization capacity, the pyruvate metabolism intermediates, stress tolerance, and virulence of this strain were characterized. Characterization of the mutant suggested that the $V$. dahliae Thit (VdThit) protein plays an important role in the pathogenicity of $V$. dahliae.

\section{RESULTS}

Deletion and complementation of VdThit in V. dahliae.

The VdThit gene in wild-type strain 991 of $V$. dahliae was replaced with a neomycin phosphotransferase resistance gene (neo) cassette that conferred neomycin resistance. Twelve transformants of $V$. dahliae 991 with neomycin resistance were analyzed by polymerase chain reaction (PCR), and five showed that the VdThit gene was replaced through homologous recombination. Two independent VdSThit deletion

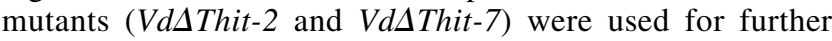
analysis (Fig. 1B and C). Additionally, to complement the VdThit gene, a functional copy of VdThit containing the promoter trpC and terminator TtrpC was successfully rein-

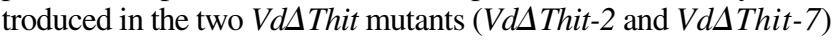
by PCR (Fig. 1D). VdThit transcription was restored in the resulting complementation strains (VdDThit-2-com and VdsThit-7-com).

A
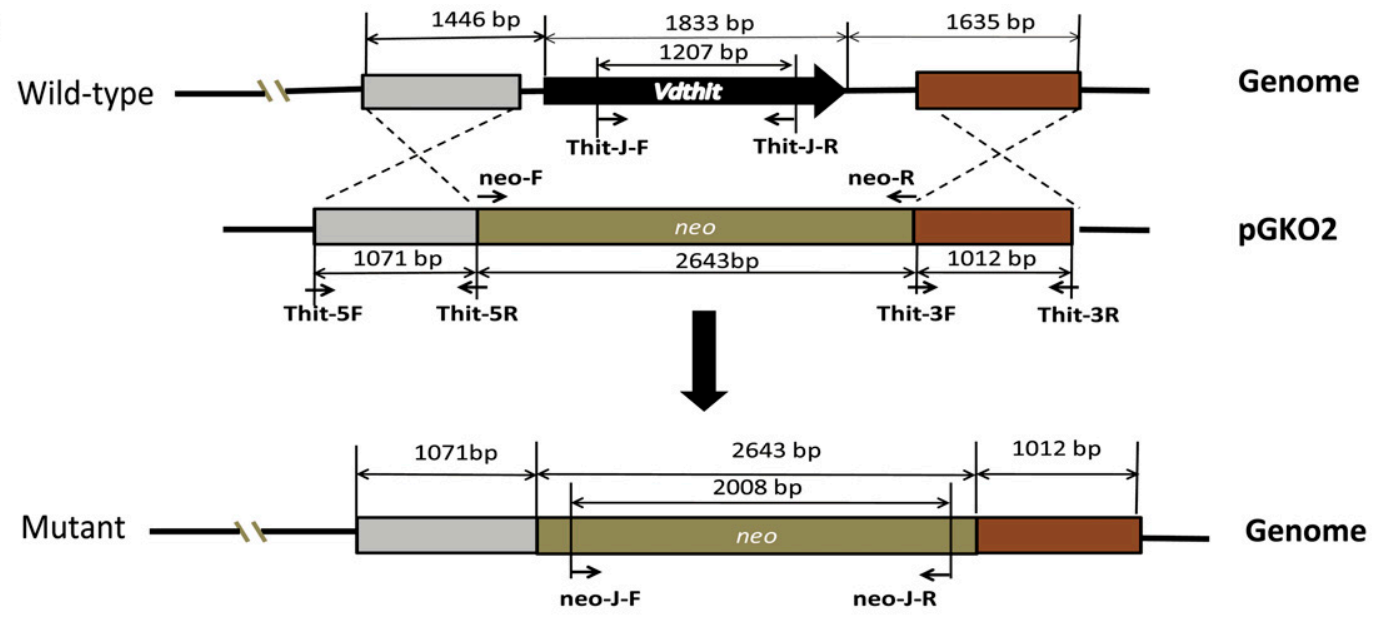

B

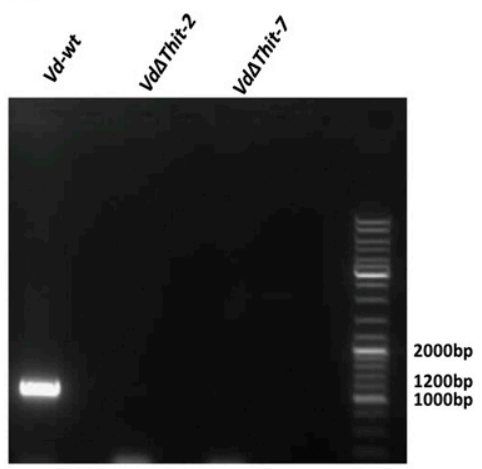

C

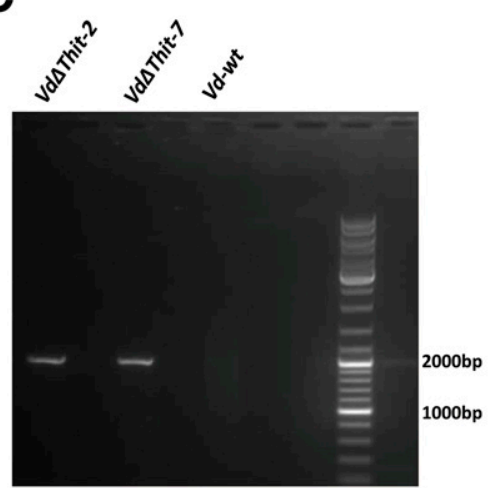

D

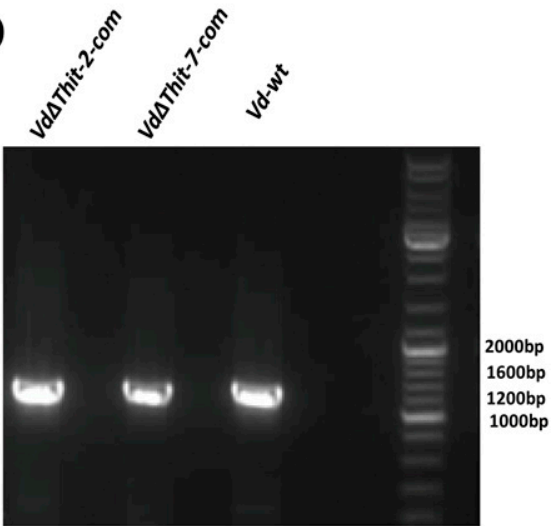

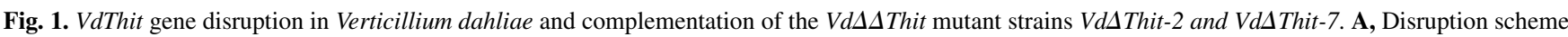
used for VdThit in the wild-type strain. B and C, Confirmation of gene disruption by polymerase chain reaction (PCR). Genomic DNA from wild type (Vd-wt) and the VdsThit mutant strains were used as templates for amplification with primer pairs Thit-J-F and Thit-J-R (B) and noe-J-F and neo-J-R(C). A 1.2-kb fragment was amplified from Vd-wt with primer pair Thit-J-F and Thit-J-R (B) and a 2.0-kb fragment was amplified using primer pair neo-J-F and neo-J-R (C)

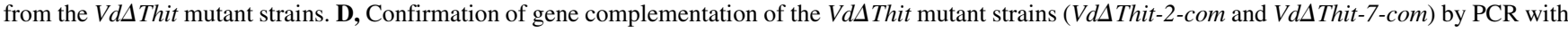
primer pair Thit-J-F and Thit-J-R; a 1.2-kb fragment was amplified. 
Mycelial radial growth, conidiation, and conidial germination were significantly reduced in the VdDThit mutants.

The role of VdThit in mycelial growth was studied, using two

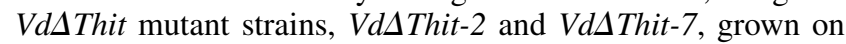
agar minimal medium (MM) with different carbon sources (sucrose, pectin, starch, galactose, or xylose) and compared with that in the $V$. dahliae wild-type strain and complementa-

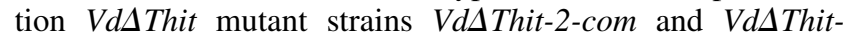
7-com. Colony diameter of the VdDThit mutants was less than that of the parental strain on MM plates with different carbon sources. Colony diameter of the VdDThit mutants on different carbon sources (sucrose, pectin, starch, galactose, and xylose) was reduced to $68,62,53,48$, and $50 \%$, respectively, of the level of the wild type (Fig. 2A and B). Likewise, conidial production of the VdSThit mutants was significantly reduced compared with the wild-type strain and complementation VdsThit mutant strains cultured on Czapek-Dox agar plates (Fig. 3A). In addition, conidia of the VdsThit mutant strains germinated more slowly than those of the wild-type strain and

A

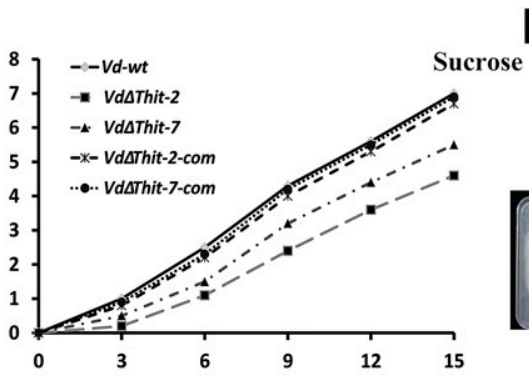

B
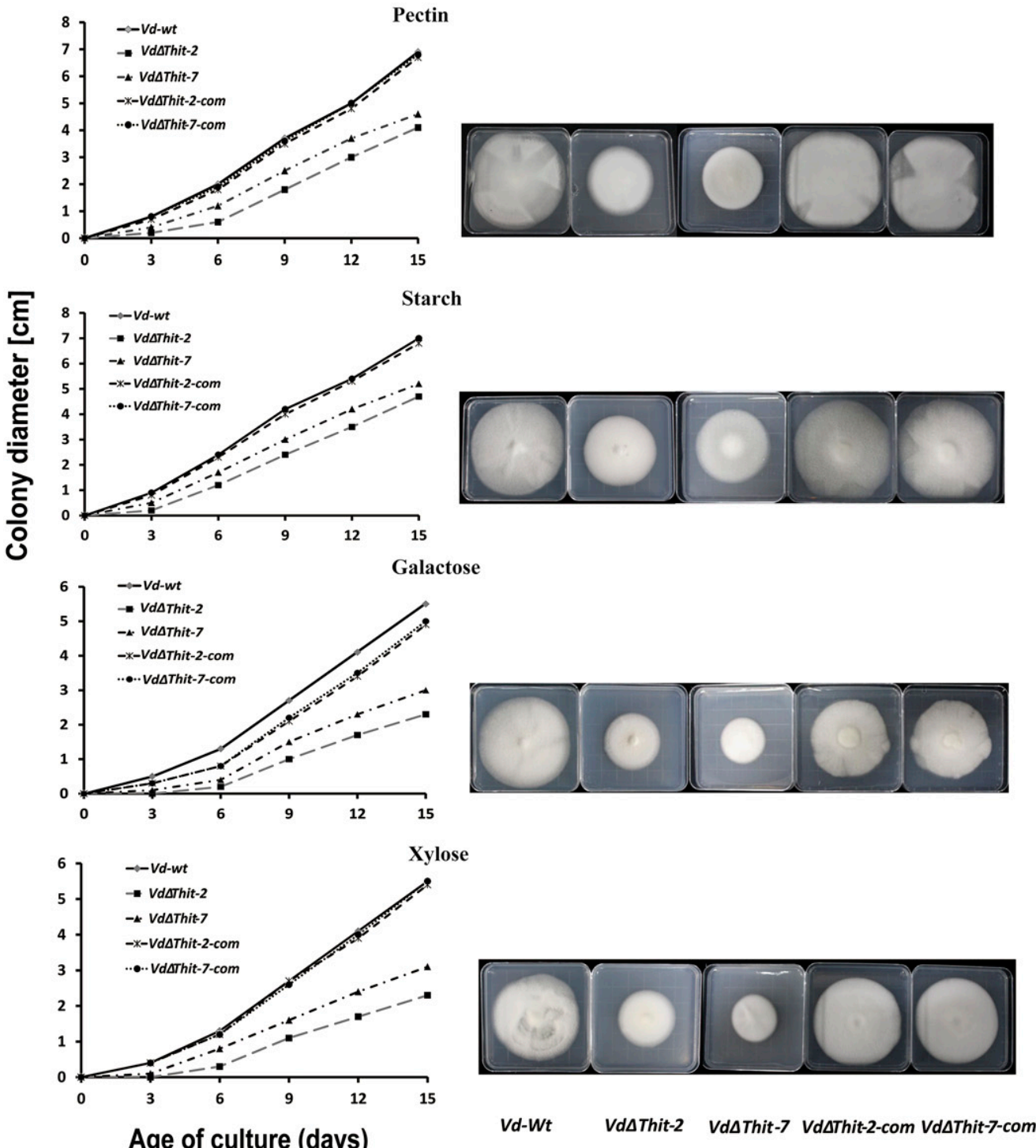

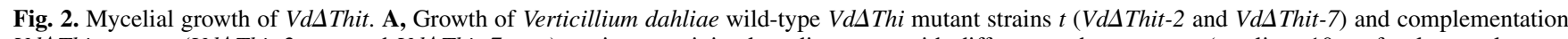

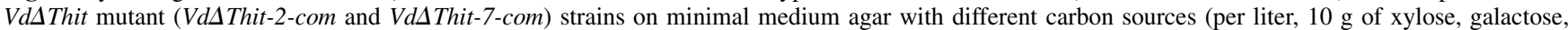
pectin, or starch or $30 \mathrm{~g}$ of sucrose). Colony diameters were measured after 3, 6, 9, 12, and 15 days. B, Colony morphology was observed at 15 days. Values represent the mean \pm standard deviation from three independent replicates. 
complementation VdsThit mutant strains; after $30 \mathrm{~h}$ of incubation in liquid complete medium (CM), only about $36 \%$ of the conidia of the VdsThit mutant strains had germinated, compared with $90 \%$ of the wild-type conidia (Fig. 3B). These results suggested that growth and development in the VdDThit mutant strains were impaired remarkably under these conditions.

\section{Loss of VdThit resulted in reduced stress tolerance.}

Thiamine plays an important role in tolerance to various abiotic stresses (Rapala-Kozik et al. 2012). To determine whether the VdThit gene was involved in abiotic stress tolerance, various types of abiotic stress (UV irradiation, oxidative stress, and osmotic stress) were tested for their effects on the relative growth inhibition of the VddThit mutant strains. To ascertain the contribution of VdThit to UV-irradiation resistance, the viability of spores was assessed with a vital strain. Significantly fewer colonies of the VdsThit mutant strains survived

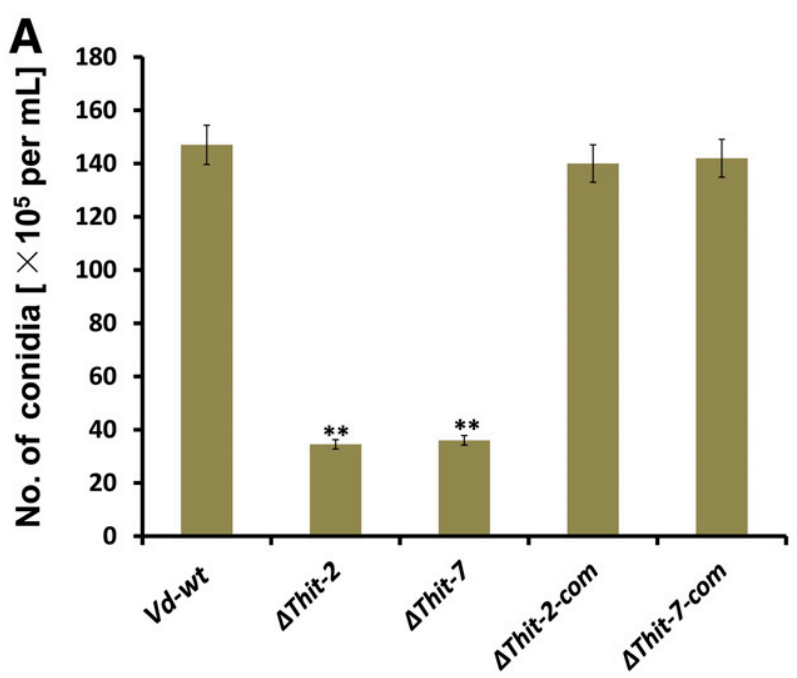

B

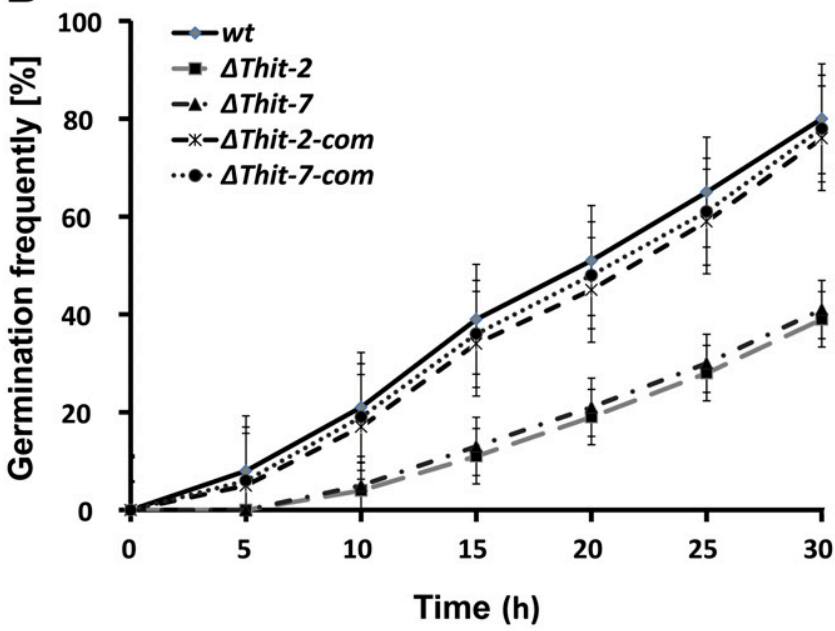

Fig. 3. Deletion of VdThit affects conidial production and germination. A, Conidial production by the Verticillium dahliae wild-type strain, VdsThit

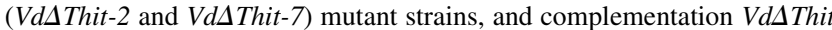

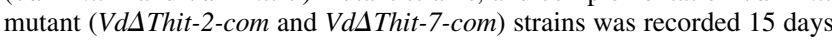
after inoculation on Czapek-Dox agar plates. B, Percentages of germinated

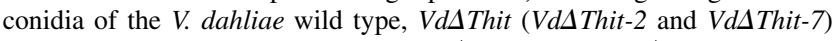

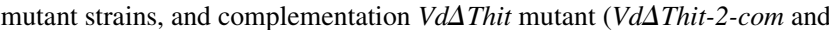
VdsThit-7-com) strains were calculated 5, 10, 15, 20, 25, and $30 \mathrm{~h}$ after inoculation in liquid complete medium. Values represent the mean \pm standard deviation from three independent replicates. Asterisks (**) indicate significant difference in Student's $t$ test at $P<0.01$. after UV exposure compared with those of the wild-type strain and complemented $V d \Delta$ Thit mutant strains $(P<0.01)$. Specifi-

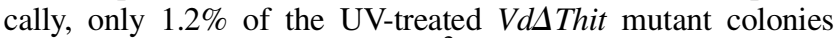
survived UV irradiation at $50 \mathrm{~J} \mathrm{~m}^{-2}$, compared with $11.5 \%$ of the wild-type colonies (Fig. 4A). Our results for VdsThit mutant strains are similar to previous studies on VdDThi4 (involved in thiazole biosynthesis of the de novo thiamine synthesis pathway) mutant strains that cannot repair damage caused by UV (Hoppenau et al. 2014).

The contribution of VdThit to strain tolerance to various stresses was investigated by growing the Vd $\Delta$ Thit mutant strains, wild-type strain, and complemented strains on Czapek-Dox agar plates with $10 \mu \mathrm{g}$ of menadione per liter (oxidative stress) or $1 \mathrm{M}$ sorbitol (osmotic stress) and comparing subsequent growth of the strains. The VdSThit mutant strains grew much slower than the

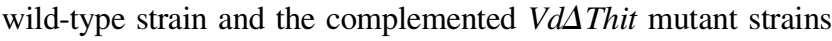
on menadione (Fig. 4B and C). However, when the $V d \Delta$ Thit mutant strains were compared with $V$. dahlia wild-type strains on sorbitol, the growth of all strains was inhibited to an equivalent degree (Supplementary Fig. S1). In short, our results showed that the VddThit mutant strains were more susceptible to oxidative stress than the other strains (Fig. 4).

\section{VdThit was required for virulence.}

We then assessed the role of VdThit in virulence of the $V d \Delta$ Thit mutant strains in Nicotiana benthamiana by dipping intact roots in a conidial suspension of the wild-type strain, the complementation $V d \Delta$ Thit mutant strains, or the VdsThit mutant strains. Interestingly, after 8,10 , and 12 days postinoculation (dpi), the wilting phenotype was significantly reduced in plants inoculated with the VdSThit mutant strains compared with the wild-type strain and complementation VdDThit mutant strains. The plants inoculated with the wild-type strain or complemented $V d \Delta$ Thit mutant strains exhibited typical symptoms, including stunting, chlorosis, wilting, and death, at 12 dpi. In contrast, those inoculated with the VdsThit mutant strains showed only a mild interveinal chlorosis of leaves, which never became necrotic (Fig. 5A and C). Fewer fungal colonies grew from excised stem tissue sections of plants inoculated with the VdsThit mutant strains than with the wild-type strain or the complemented $V d \Delta T h i t$ mutant strains (Fig. 5B). Furthermore, less fungal DNA was amplified from infected plants inoculated with the VdAThit mutant strains than with the wild-type strain or the complemented $V d \Delta T h i t$ mutant strains (Fig. 5D). These results suggested that the VdsThit mutant strains were defective in virulence.

\section{Pyruvate metabolism intermediates in the VdsThit mutant strains decreased, as shown by mass spectrometry analyses.}

Because the cofactor thiamine diphosphate TPP is required for the activity of pyruvate decarboxylase in the Krebs cycle and for pyruvate dehydrogenase during pyruvate metabolism (Lu et al. 2000; Moe et al. 1974; Mojzita and Hohmann 2006), we used gas chromatography-mass spectrometry (GC-MS) and high-pressure liquid chromatography-mass spectrometry (HPLCMS) to ascertain whether the lowered virulence and the stress phenotypes of the $V d \Delta$ Thit mutant strains and the depletion of TPP were also associated with restricted pyruvate dehydrogenase and pyruvate decarboxylase by analyzing the Krebs cycle and pyruvate metabolism intermediates. These analyses indicated that acetoin in pyruvate metabolism (Fig. 6) and acetylcoenzyme A (acetyl-CoA) in the Krebs cycle were significantly reduced in the $V d \Delta$ Thit mutant strains compared with those in the V. dahliae wild type (Fig. 7). We thus concluded that the TPP depletion in the $V d \Delta$ Thit mutant strains was confined to the Krebs cycle and pyruvate metabolism, restricting their energy production and leading to the defective virulence and stress phenotypes. 
Exogenous thiamine partially restored vegetative growth, conidiation, and virulence of the VdDThit mutants.

Our earlier experiments showed the VdsThit mutant strains grew noticeably slower than the wild type on MM agar plates. When supplemented with 50 to $1,000 \mu \mathrm{g}$ of exogenous thiamine

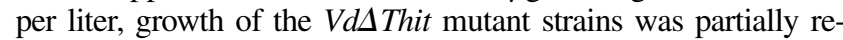
stored to the level of the wild type (Fig. 8A and B). To determine whether exogenous thiamine could restore the defective conidiation of the VddThit mutants, the Czapek-Dox agar plates were supplemented with 50 to $1,000 \mu \mathrm{g}$ of exogenous thiamine per liter. When cultured with exogenous thiamine, the VdsThit mutant strains produced significantly more conidia than on the unsupplemented with exogenous thiamine Czapek-Dox agar plates but not at the level of the wild type (Fig. 8C).
To examine the virulence of the recovered conidia, we inoculated $N$. benthamiana roots with a suspension of conidia collected from the colonies grown on Czapek-Dox agar plates with exogenous thiamine $(0,1$, and $50 \mu \mathrm{g} / \mathrm{liter})$. As shown in Figure 8D, at $12 \mathrm{dpi}$, the virulence defect was partially overcome in $V d \Delta T h i t$ mutant strains grown from spores from the medium with $50 \mu \mathrm{g}$ of thiamine per liter (Fig. 8D). Similarly, in the $\Delta$ Moilv1 mutant of Magnaporthe oryzae, a disruptant of MoILV1, vegetative growth was fully restored and conidiation and virulence were partially restored on MM supplemented with exogenous isoleucine ( $\mathrm{Du}$ et al. 2014). These results strongly suggest that VdThit contributes to thiamine absorption and transport during vegetative growth, development, and infection.
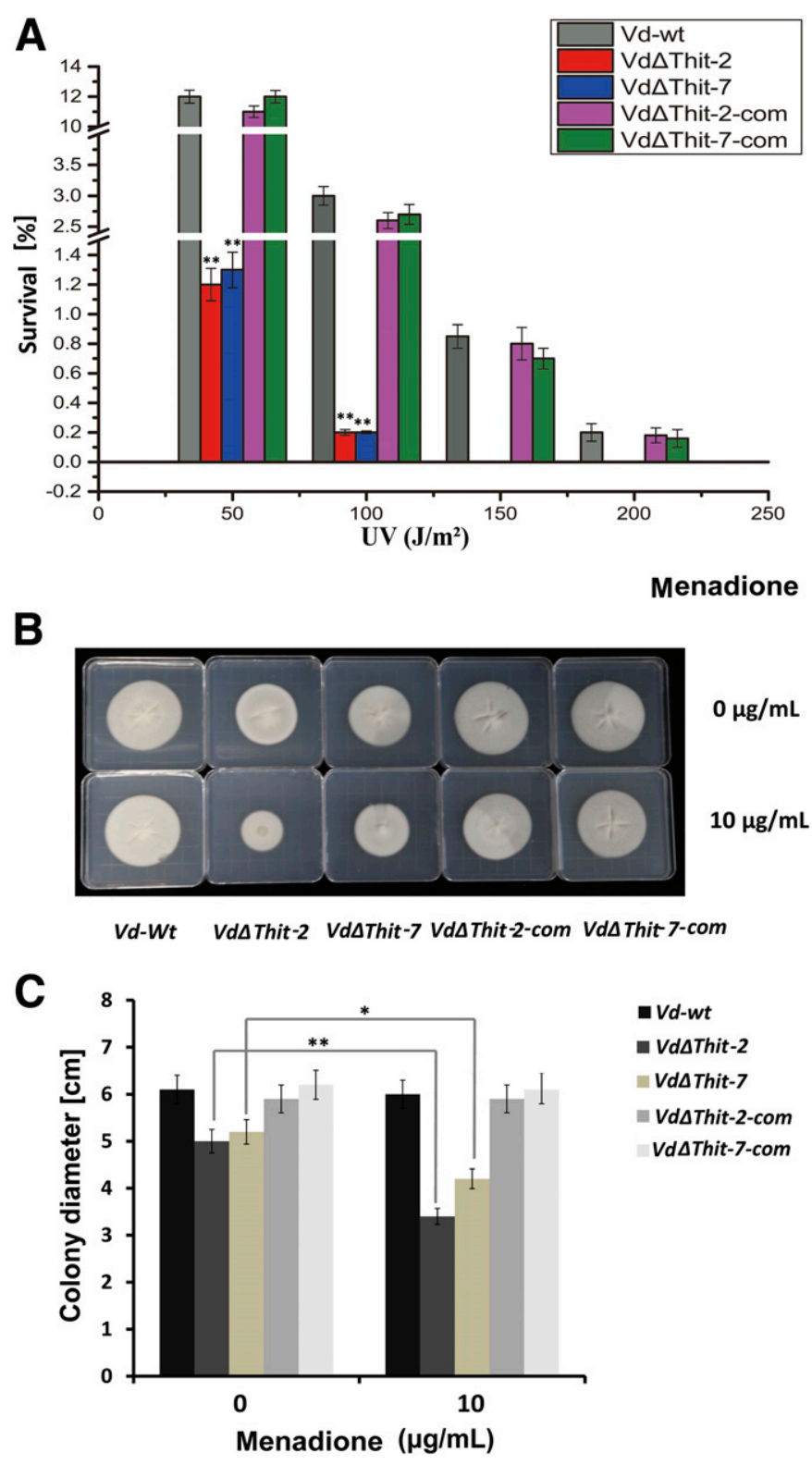

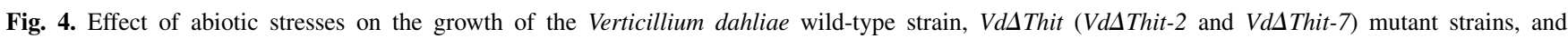

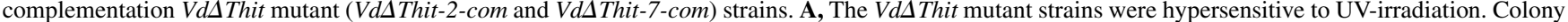

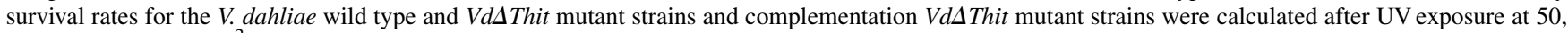

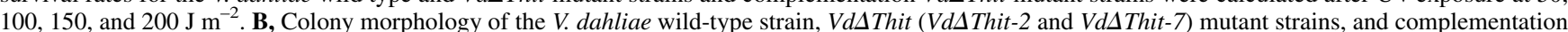

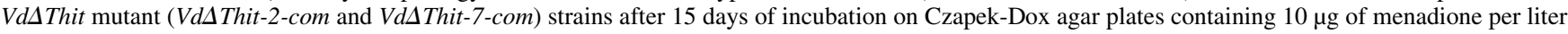
compared with no-stress controls at $25^{\circ} \mathrm{C}$. C, Colony diameters at 15 days after inoculation on Czapek-Dox agar plates with $10 \mu \mathrm{g}$ of menadione per liter compared with no-stress controls. Values represent the mean \pm standard deviation from three independent replicates. Asterisks (**) indicate significant difference in Student's $t$ test at $P<0.01$. 
Upregulation of de novo thiamine synthesis genes in the VdDThit mutant.

To confirm that VdThit plays an important role in thiamine biosynthesis, transport, and usage, we first focused on regulation by profiling the transcripts of the VdSti35 and Vdthill genes from the de novo thiamine synthesis pathway, using quantitative reverse transcription (qRT)-PCR analyses of cDNA samples from 7-day-old cultures of the $V d \Delta$ Thit mutant strains, wild-type strain, and complemented VdDThit mutant strains. In the VdsThit mutant strains, VdSti35 and Vdthill gene transcript levels were five- and sixfold higher, respectively, than in the wild-type strain and complemented VdDThit mutant strains (Fig. 9A and C). In liquid CM supplemented with 50 to $1.000 \mu \mathrm{g}$ of exogenous thiamine per liter, transcription of the VdSti35 and

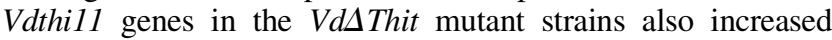
compared with the wild-type strain and complemented $V d \Delta$ Thit mutant strains in the same medium and compared with the levels of the respective $V d \Delta$ Thit mutant strains in liquid CM without
A
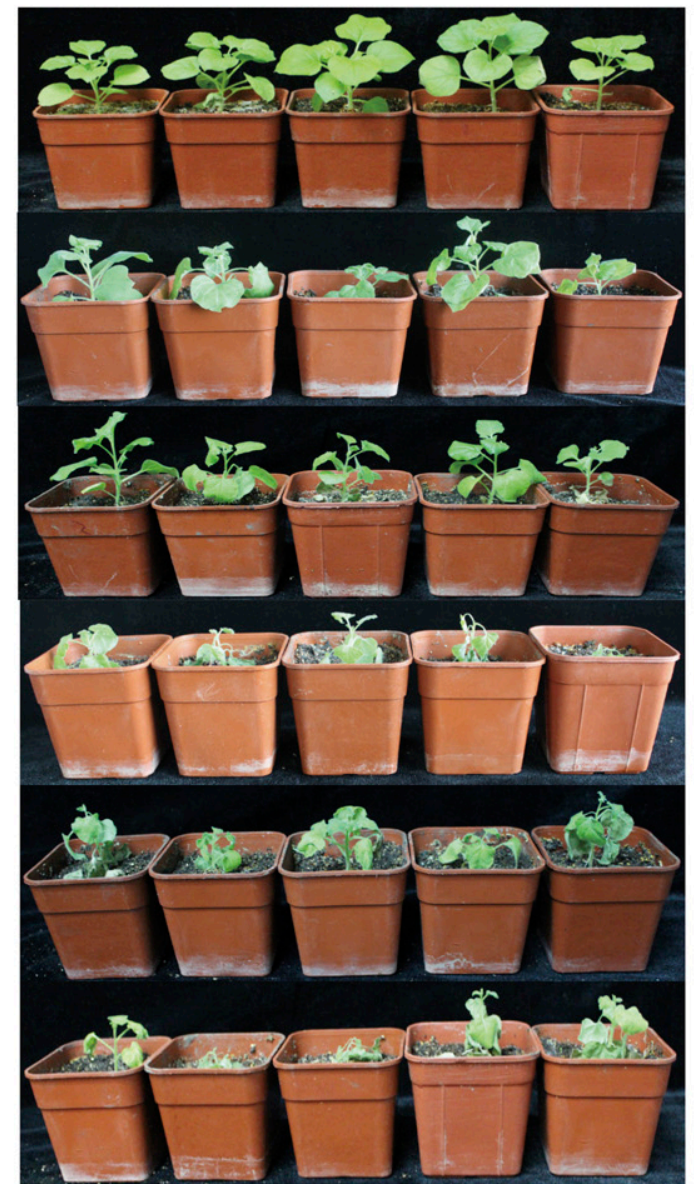

$\sqrt{2}$

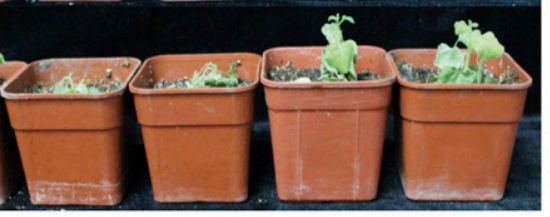

C

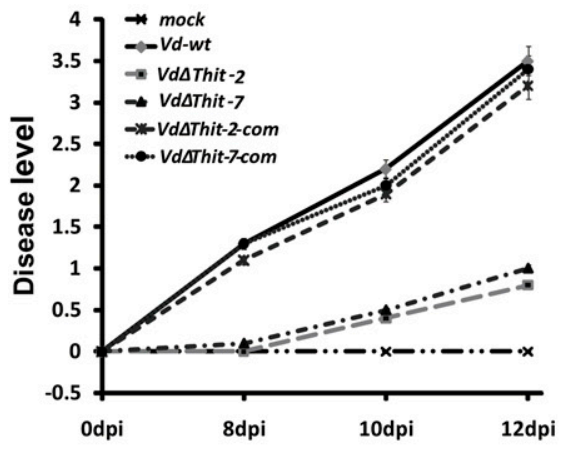

B

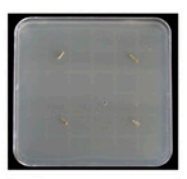

Mock

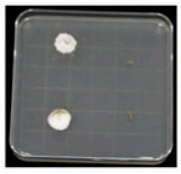

VdSThit -2

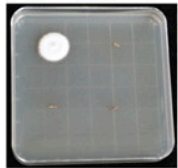

VdSThit -7

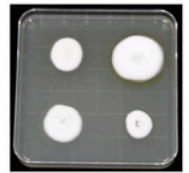

$V d-W t$

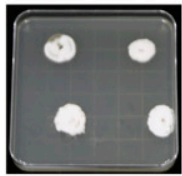

VdSThit -2-com

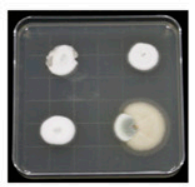

VdaThit -7-com
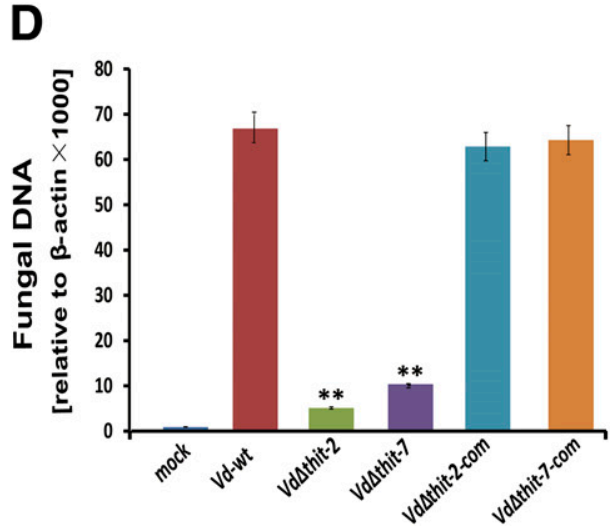

Fig. 5. VdThit was required for virulence of Verticillium dahliae on Nicotiana benthamiana. A, Representative images of plants infected with the V. dahliae

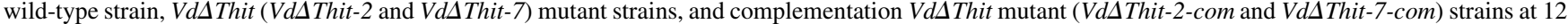
days postinoculation (dpi). B, Fungal regrowth on potato dextrose agar 7 days after incubation of plant stem tissue sections excised at 12 dpi. C, Disease level of

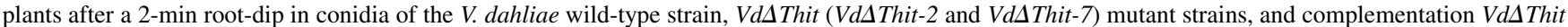

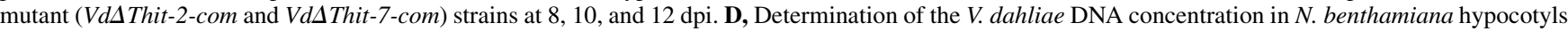

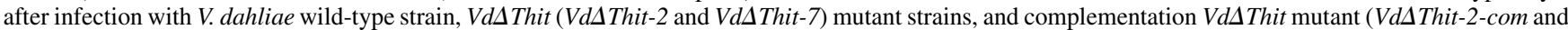
VdsThit-7-com) strains at 12 dpi. Tap water-inoculated plants (mock) as a control did not yield any V. dahliae DNA. Values represent the mean \pm standard deviation from three independent replicates. Asterisks (**) indicate significant difference in Student's $t$ test at $P<0.01$. 
exogenous thiamine (Fig. 9A and C). In short, our results showed that de novo thiamine synthesis genes were upregulated in the $V d \Delta$ Thit mutants, suggesting that thiamine synthesis de novo compensated for the shortage of thiamine when the thiamine transport system was not functional and was also regulated by the addition of thiamine. Thus, we speculate that VdThit may play another important role in V. dahlia.

\section{Root infection and fungal colonization} by the $V d \Delta$ Thit mutant were impaired.

To analyze the defect in infection and colonization of plant root tissues of the $V d \Delta$ Thit mutant strains, we used VdDThit-7-GFP and $V d-w t-G F P$ transformant strains that constitutively express enhanced green fluorescent protein (eGFP), to enable observation of roots after inoculation with the VdDThit-GFP mutant strain or $V d-w t-G F P$ strain by laser scanning confocal microscopy (LSCM). First, there was no difference in growth, development, or virulence between the wild-type strain and $V d-w t-G F P$ strain or between the $V d \Delta$ Thit mutant strain and VdDThit-GFP strain (Supplementary Fig. S2). Roots of $N$. benthamiana seedlings at the third leaf stage were dipped into a conidial suspension of $V d \Delta$ Thit-7-GFP strain or $V d-w t-G F P$ strain to monitor infection and fungal colonization. By $24 \mathrm{~h}$ postinoculation (hpi), germ tubes and hyphae from $V d-w t-G F P$ strain conidia had elongated extensively at random positions on the $N$. benthamiana root surface- and hyphae had elongated and surrounded the root (Fig. 10). In contrast, Vd $\Delta$ Thit-7-GFP strain conidia formed germ tubes but they did not elongate, so no hyphae developed (Fig. 10). By 7 dpi, strains $V d \Delta T h i t-7-G F P$ and $V d-w t-G F P$ had colonized the vascular system of the roots, but hyphae of the $V d$-wt-GFP strain had proliferated within the vascular tissue in contrast to a small hyphal net in xylem vessels infected with
VdDThit-7-GFP strain (Fig. 10). The VdAThit mutant was thus defective in the infection and colonization of plant root tissues.

The VdThit gene of $V$. dahliae plays an important role in vegetative development and invasive hyphal growth as shown by qRT-PCR.

The thiamine transporter is expressed ubiquitously in various tissues of Saccharomyces cerevisiae (Enjo et al. 1997; Singleton 1997). For analyzing the expression pattern of VdThit during spore germination, hyphal growth, and conidiogenesis of $V$. dahliae on media and in infected roots of $N$. benthamiana, RNA was extracted from conidia or hyphae harvested after $0,3,6,9,12,24,72,96$, and $120 \mathrm{~h}$ of growth in liquid CM for qRT-PCR. VdThit expression was highest during late hyphal growth at $48 \mathrm{~h}$ and was also higher during conidiogenesis than during conidial germination (Fig. 11A). In infected $N$. benthamiana, VdThit expression was low during early stages of disease ( 0 to 3 days) with the lowest expression at 1 to 2 days, even lower than in harvested conidia (Fig. 11B). From 4 to 8 days, VdThit expression was higher than in harvested conidia and was highest at 5 to 6 days (Fig. 11B). This experiment suggested that the VdThit gene plays an important role in vegetative growth and reproductive development and in invasive hyphal growth.

\section{DISCUSSION}

In the present study, we analyzed the biological function of the V. dahliae Thit gene. Deletion of VdThit resulted in reduced vegetative growth, conidial production, stress-response capacity, and invasive growth and colonization of plant hosts. Interestingly, the defects in vegetative growth, conidiation, and stress responses of the VdSThit mutant were accompanied by
A

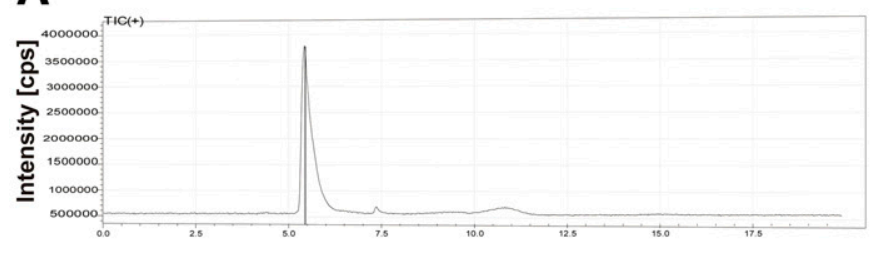

C Time(min)

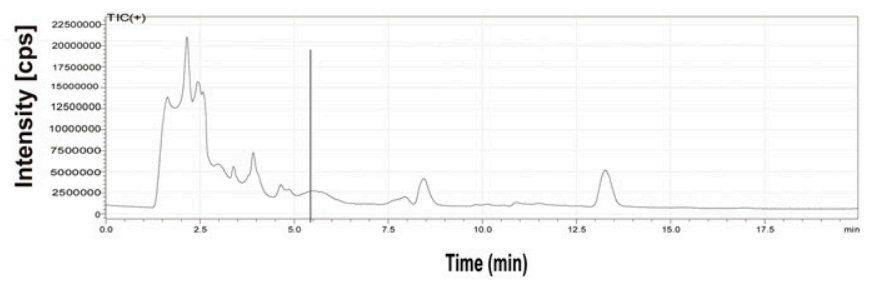

E

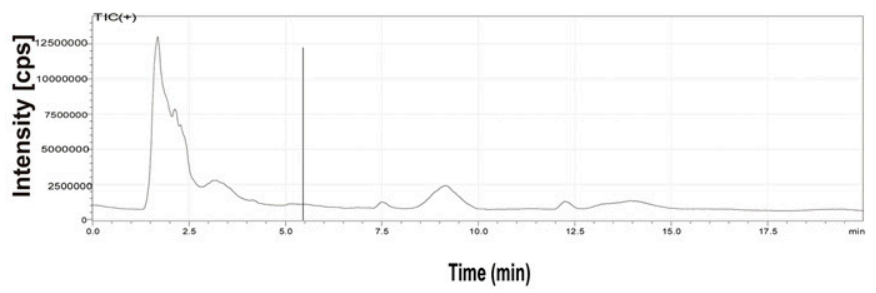

B
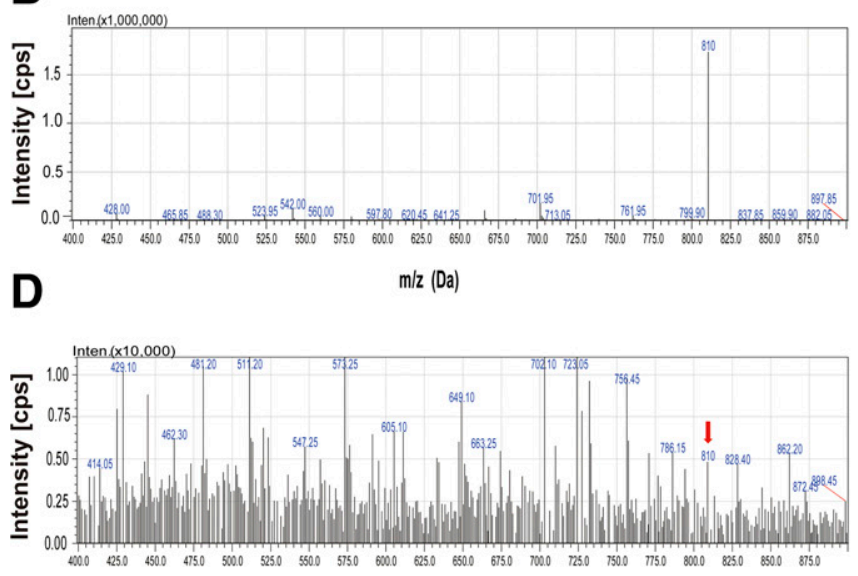

$\mathrm{m} / 2$ (Da)

$\mathbf{F}$

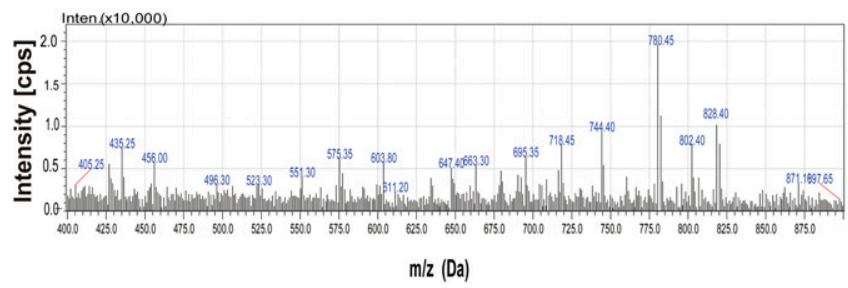

Fig. 6. High-pressure liquid chromatography-mass spectrometry (MS) analyses of acetyl-CoA in the Krebs cycle of VdsThit mutant strains and wild-type strain. A, Multiple-reaction monitoring (MRM) chromatograms of acetyl-CoA standard solution and B, MS spectrum (precursor ion scan of $810 \mathrm{~m} / \mathrm{z}$. C, MRM chromatograms of acetyl-CoA in extracts from mycelium of wild-type strain cultivated for 5 days and $\mathbf{D}$, MS spectrum (precursor ion scan of $810 \mathrm{~m} / \mathrm{z}$ ) at retention time of $5.53 \mathrm{~min}$. E, MRM chromatograms in extracts from mycelium of Vd $\Delta$ Thit mutant strains cultivated for 5 days and $\mathbf{F}$, MS spectrum (precursor ion scan of $810 \mathrm{~m} / \mathrm{z}$ ). cps $=$ counts per second. 
greatly impaired virulence. Our results provide evidence that VdThit is required for the pathogenicity of $V$. dahliae.

In previous research, VdThi4 (Vdsti35, a gene of the de novo thiamine synthesis pathway) was found to be required for $V$. dahliae infection of tomato and might contribute to the oxidative-stress response but be unrelated to osmotic stress (Hoppenau et al. 2014). However, thiamine uptake and transport regulation as well as the contribution of the stress response to species pathogenicity was not examined. The Fusarium oxysporum ortholog of Vdsti35, Fosti35, did not influence virulence, and an ambient thiamine supply was suggested to be necessary for this fungus to grow (Ruiz-Roldán et al. 2008). Nevertheless, our study showed that growth rate, conidiation, and stress responses (oxidative stress and osmotic stress) of the $V d \Delta$ Thit mutants were significantly reduced compared with the $V$. dahliae wild type. We speculate that uptake of exogenous thiamine by the thiamine transporter protein is the main way that $V$. dahliae obtains thiamine. In $S$. cerevisiae, a thiamine transporter (thi7) serves as a high-capacity, primary uptake system for the bulk of the thiamine acquired (Mojzita and Hohmann 2006). In S. cerevisiae, Yor71c and Yor192c, which encode proteins that contribute to thiamine uptake, have a low capacity and low affinity for thiamine. And in the $V$. dahliae Ls.17 genome (Verticillium Comparative Genomics Project database) (Klosterman et al. 2011), there are no paralogs of Yor71c and Yor192c.
When exogenous thiamine is low or absent, the expression of several THI genes (de novo thiamine synthesis) is strongly upregulated to synthesize thiamine for organism use (Hohmann and Meacock 1998; Mojzita and Hohmann 2006). Similarly, deletion of VdThit resulted in the upregulation of Vdsti35 and VdThil1 expression for de novo thiamine synthesis. And when the VdsThit mutant strains were supplemented with high exogenous thiamine, the de novo thiamine synthesis genes (Vdsti35 and VdThil1) were more strongly upregulated when VdThit had been deleted (Mojzita and Hohmann 2006). In Schizosaccharomyces pombe, thiamine transport activity is regulated by extracellular thiamine (Iwashima et al. 1979) and the expression of THI7, which encodes a thiamine transporter protein, is induced when extracellular levels of thiamine increase to $1.2 \mu \mathrm{M}$ per liter. Some exogenous thiamine is transferred into cells by a nonsaturable or passive diffusion mechanism when the extracellular concentration of thiamine is high in S. cerevisiae (Rindi 1992; Singleton 1997), and de novo thiamine synthesis genes are then up-regulated to meet the needs of the fungus. In our study of the $V d \Delta$ Thit mutant, high supplemented levels of exogenous thiamine did not completely restore vegetative growth, conidiation, and virulence to the wild-type levels. We thus suggest that VdThit plays an irreplaceable role in the growth, development, and virulence of $V$. dahliae.

In fungi, various genes are up- or down-regulated to meet the requirements of different stages of growth and development
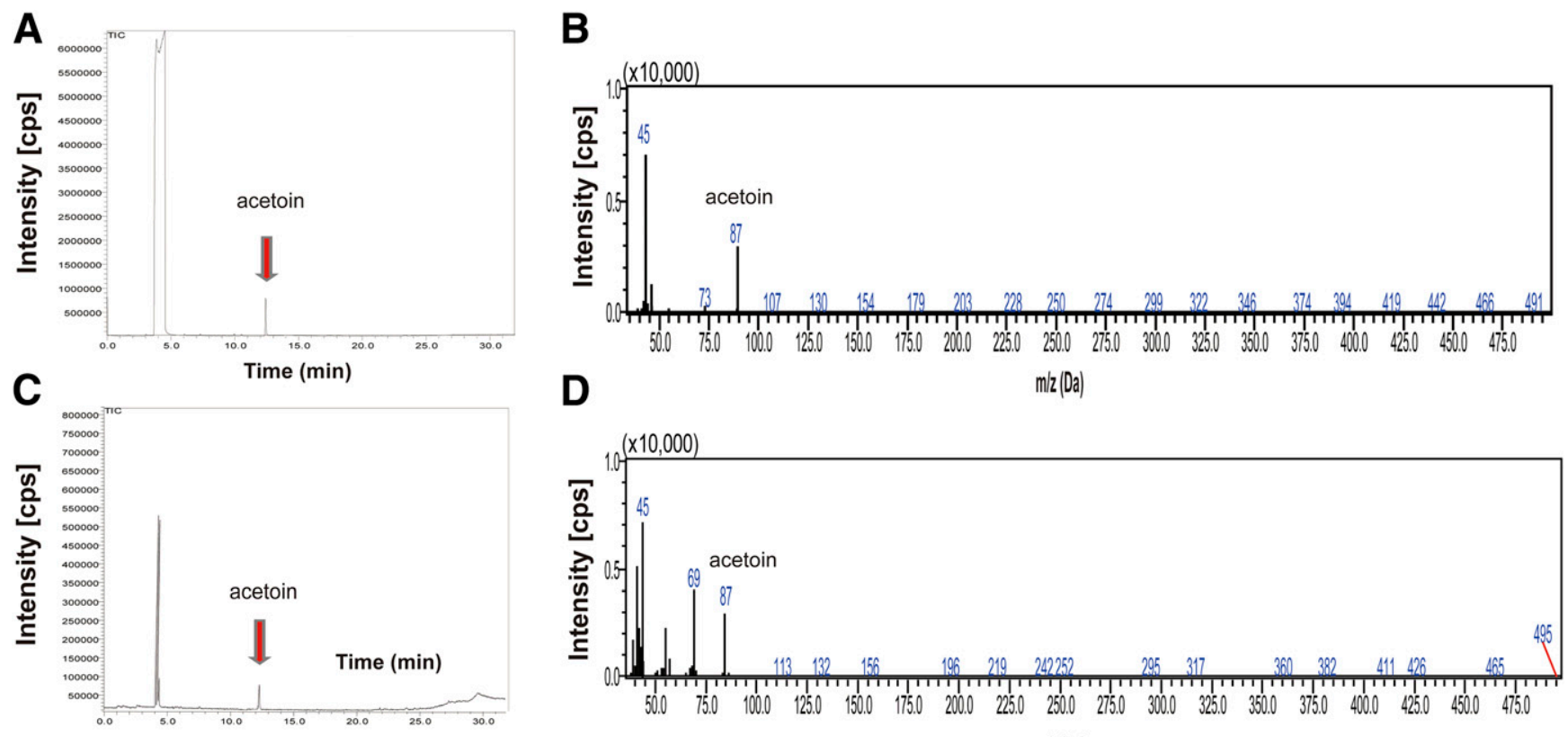

D
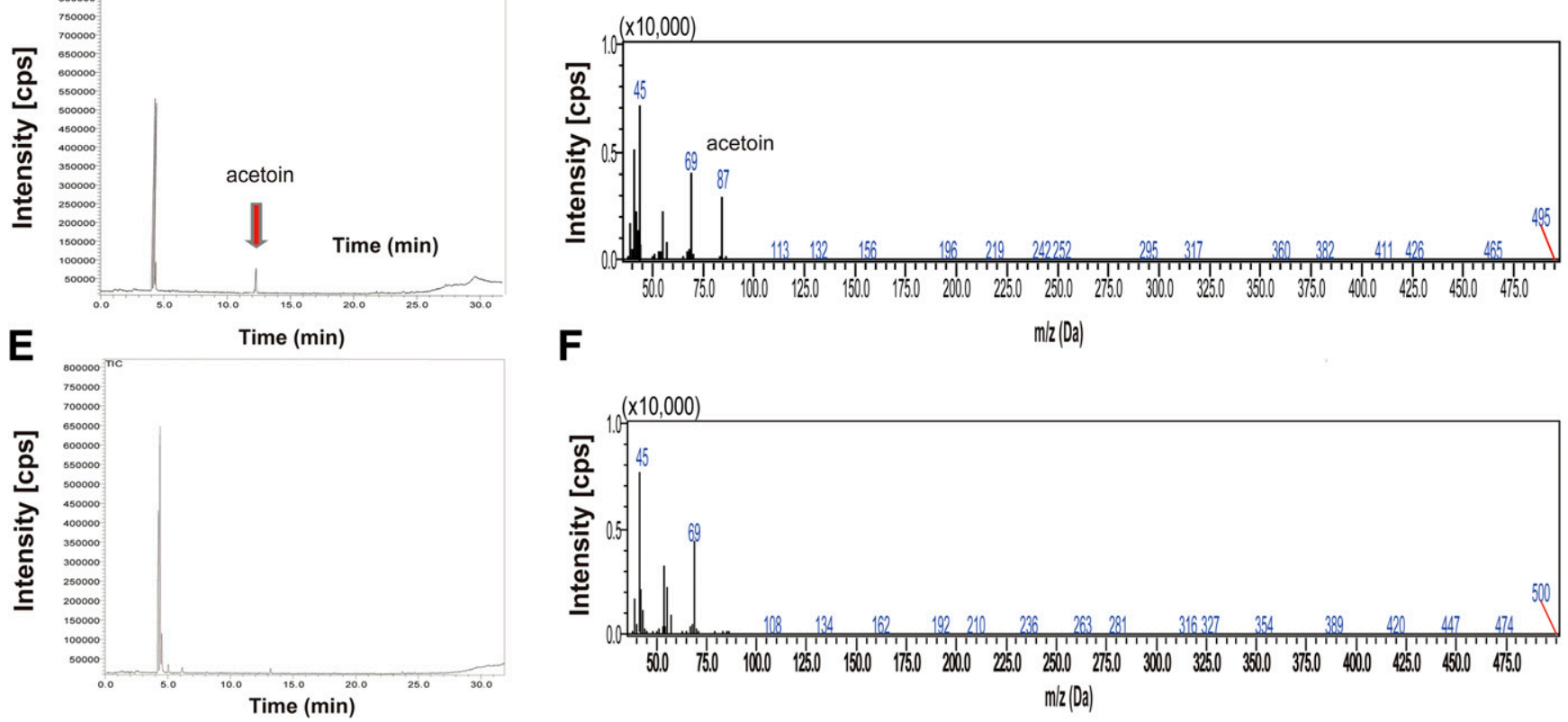

Fig. 7. Gas chromatography-mass spectrometry analyses of acetoin in pyruvate metabolism of VdDThit mutant strains and wild-type strain. A, Gas chromatograms of acetoin standard solution (ethanol solvent) and $\mathbf{B}$, mass spectrum (precursor ion scan of $87 \mathrm{~m} / \mathrm{z}$ ). $\mathbf{C}$, Gas chromatograms of acetoin in extracts from mycelium of wild-type strain cultivated for 5 days and D, mass spectrum (precursor ion scan of $87 \mathrm{~m} / \mathrm{z}$ ) at retention time of $5.53 \mathrm{~min}$. E, Gas chromatograms in extracts from mycelium of VdDThit mutant strains cultivated for 5 days and $\mathbf{F}$, mass spectrum. cps $=$ counts per second. 
(Gomes et al. 2016; Ikegami et al. 2000; Lee et al. 2008; Schumacher et al. 2016; Zeier et al. 2015). For instance, adapting to different hosts and stages of growth and development, Fusarium graminearum expressed distinct sets of genes associated with transport proteins and secondary metabolism during infection of wheat, barley, and maize (Harris et al. 2016). Genes for transport proteins and secondary metabolism were also up- or down-regulated to meet the requirements of

A

$V d-w t$

VdsThit-7

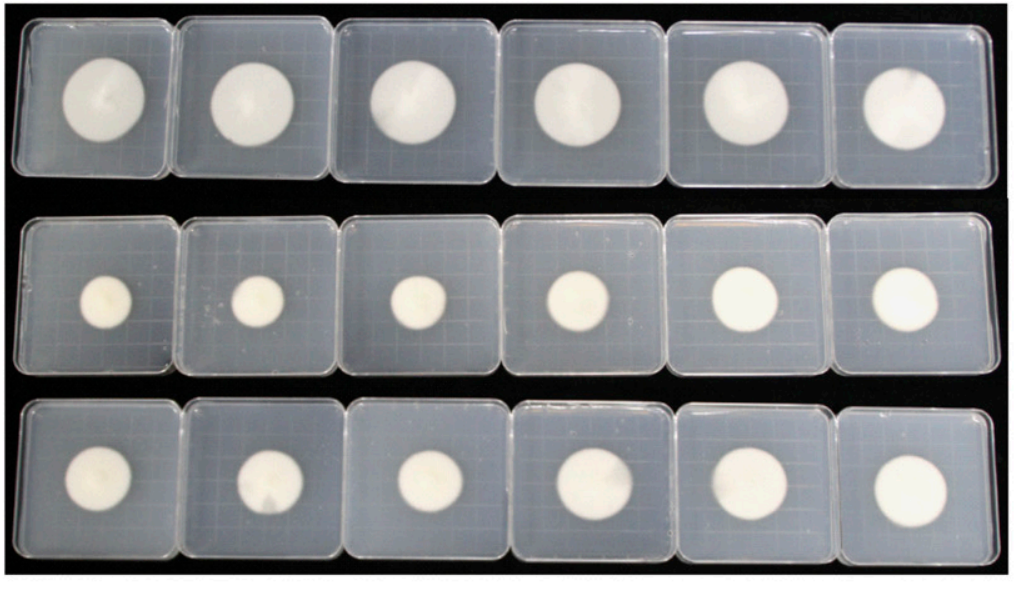

0.1

Thiamine $(\mu \mathrm{g} / \mathrm{L})$

100

1000

B

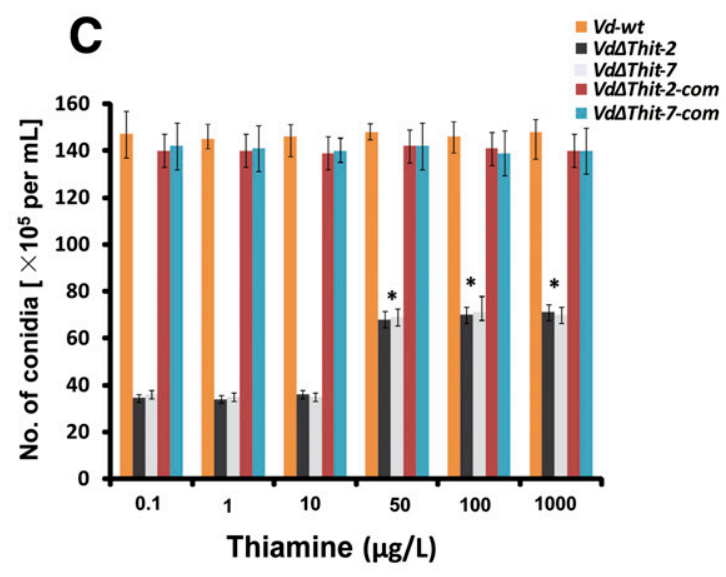

D

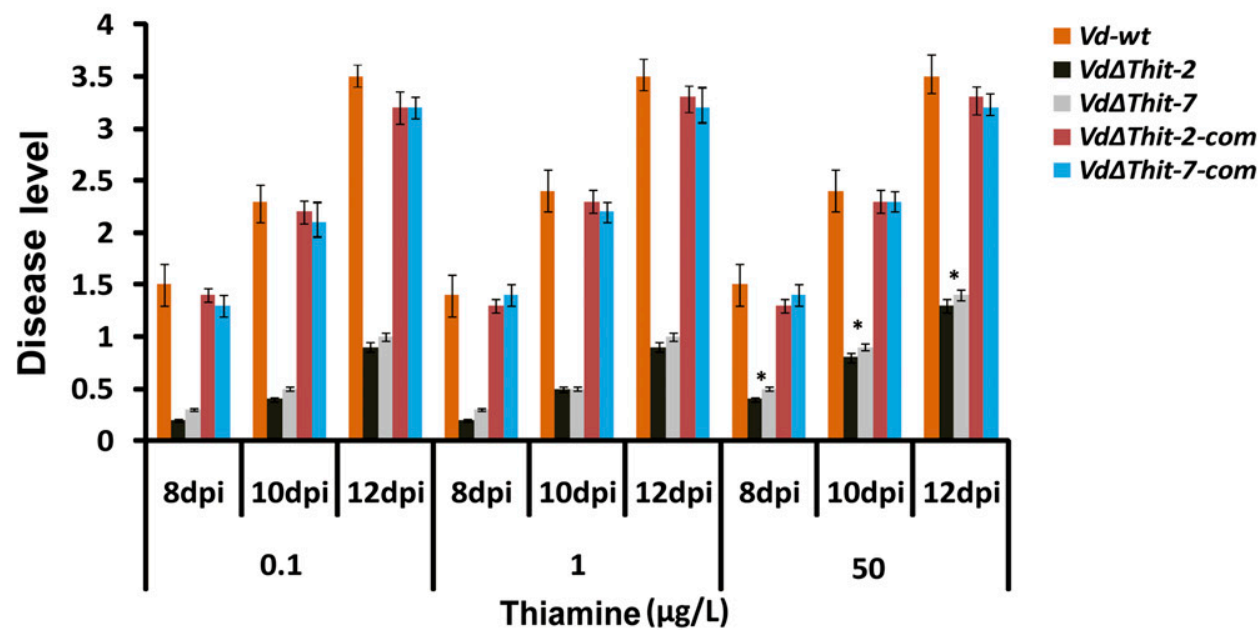

Fig. 8. Exogenous thiamine could restore the growth, conidiation, and virulence in defective Vd $\Delta$ Thit mutant strains on Czapek-Dox agar plates. A, Colony

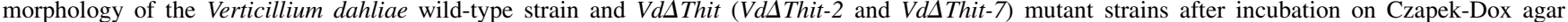
supplemented with exogenous thiamine (from 0 to $1,000 \mu \mathrm{g}$ per liter) for 7 days at $25^{\circ} \mathrm{C}$. B, Colony diameters and $\mathbf{C}$, conidial production of the V. dahliae wild-

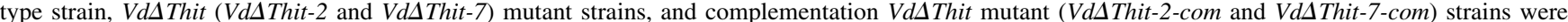
measured 7 days after inoculation. D, Disease level of Nicotiana benthamiana plants at 8, 10, and 12 days postinoculation (dpi) after a 2-min root-dip in conidia

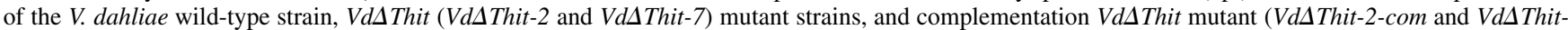
7-com) strains grown on Czapek-Dox agar supplemented with exogenous thiamine $\left(0,1\right.$, or $50 \mu \mathrm{g}$ per liter) for 7 days at $25^{\circ} \mathrm{C}$. Values are means \pm standard deviation from three replications. Asterisks (*) indicate significance in Student's $t$ test at $P<0.05$. 
different stages of growth and development (Harris et al. 2016). In Magnaporthe oryzae, an imparity in the temporal expression of different chitin synthase genes during hyphal growth, conidiogenesis, appressorium development, and plant infection processes indicated that these genes were indispensable for growth, development, and plant infection (Kong et al. 2012). In our study of VdThit expression profiles at different developmental stages, expression of VdThit was elevated during vegetative growth and conidiogenesis on $\mathrm{CM}$ and during infection of $N$. benthamiana, suggesting that VdThit may play a role in growth and development and in plant infection. We speculate that the expression of VdThit may be regulated to help the fungus adapt to external conditions at different stages of growth and development and infection of its host (CallejasNegrete et al. 2015). In addition, the continued expression of VdThit during various developmental and plant infection processes confirmed the necessity of VdThit for $V$. dahliae growth, development, and plant infection.

Just as thiamine (vitamin B1) is essential for humans (Sriram et al. 2012), thiamine and thiamine diphosphate, the active form of thiamine, are essential for cellular metabolism in fungi and green plants. Thiamine can relieve the effects of several environmental stresses in Arabidopsis thaliana by protecting the plant from oxidative and osmotic stress damage (Ahn et al. 2005; RapalaKozik et al. 2008, 2012). Thiamine-treated rice, Arabidopsis thaliana, and vegetable crop plants showed resistance to infection by various fungal, bacterial, and viral pathogens, because the treatment induces the transient expression of pathogenesis-related genes in plant defense responses and improves resistance to abiotic stresses, such as salt and ROS (Goyer 2010). In fungi, thiamine also increases resistance to stress (Hoppenau et al. 2014; Ruiz-Roldán et al. 2008). Recently, research has confirmed that thiamine biosynthetic pathway genes (THI4 and THIO) are necessary for responses to stress conditions in $S$. cerevisiae and $V$. dahliae (Hoppenau et al. 2014; Ruiz-Roldán et al. 2008). Consistent with this research, our results showed that deletion of VdThit increased susceptibility to oxidative stress, osmotic stress, and DNA damage, suggesting that VdThit helps the fungus adapt to specific environmental conditions. Research on Beauveria bassiana suggests that the virulence defect in the $\triangle B b M B F 1$ mutant was likely due primarily to impaired hyphal morphogenesis and adaptation to specific environmental stresses (Ying et al. 2014). In Phytophthora sojae, the impaired ability of Psimpal mutants to tolerate and remove ROS resulted in decreased virulence (Yang et al. 2015). The intrinsic osmotic conditions in plant tissues can also reduce mycelial growth; we speculate that susceptibility to abiotic stress conditions may be responsible for the virulence defect in the VdsThit mutant (Shao et al. 2015).

The thiamine diphosphate TPP, the active form of thiamine, as a key cofactor of pyruvate decarboxylase and pyruvate dehydrogenase, plays an important role in the metabolism of pyruvate (Lu et al. 2000; Moe et al. 1974; Mojzita and Hohmann

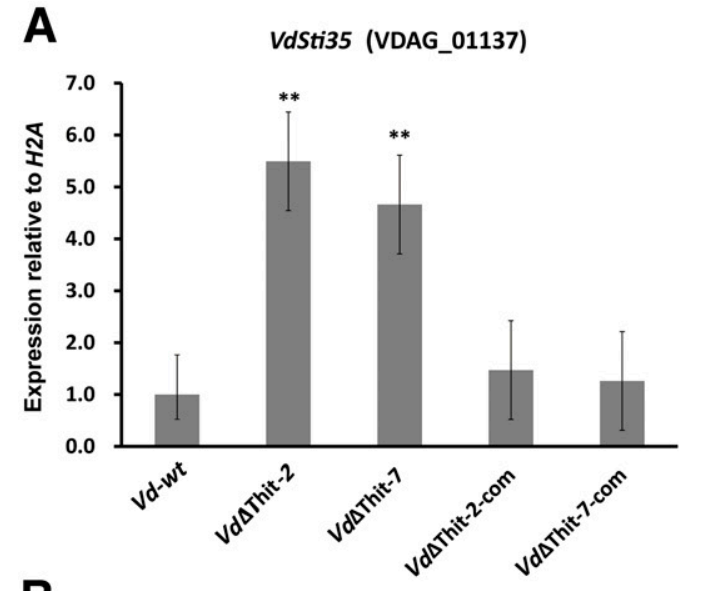

B

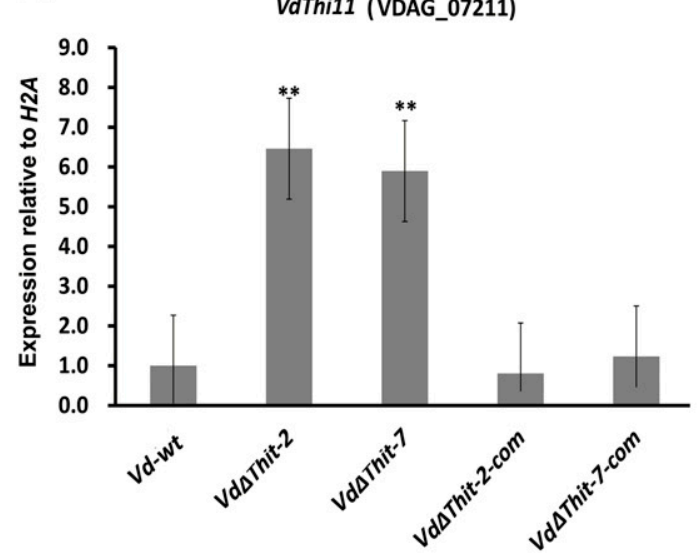

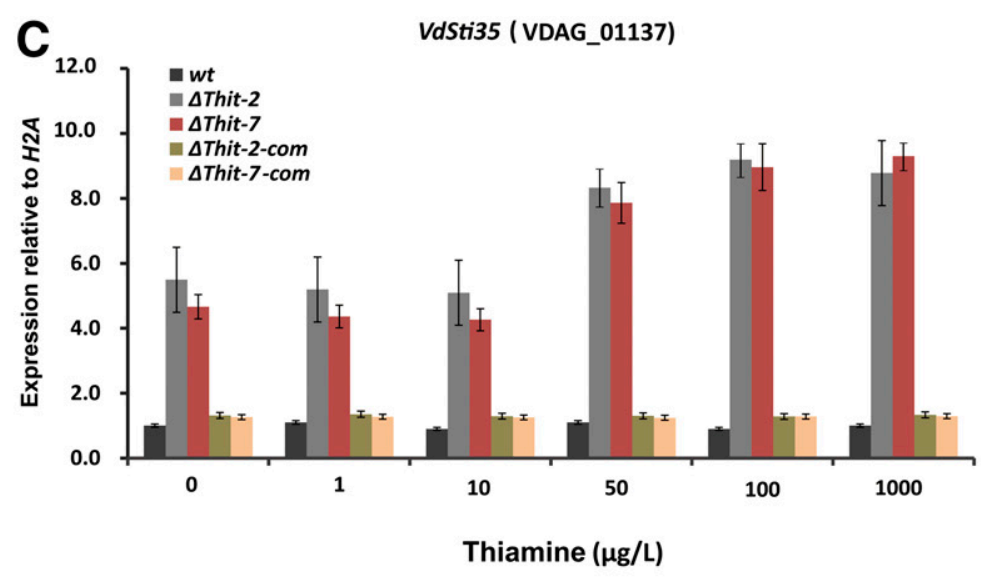

D

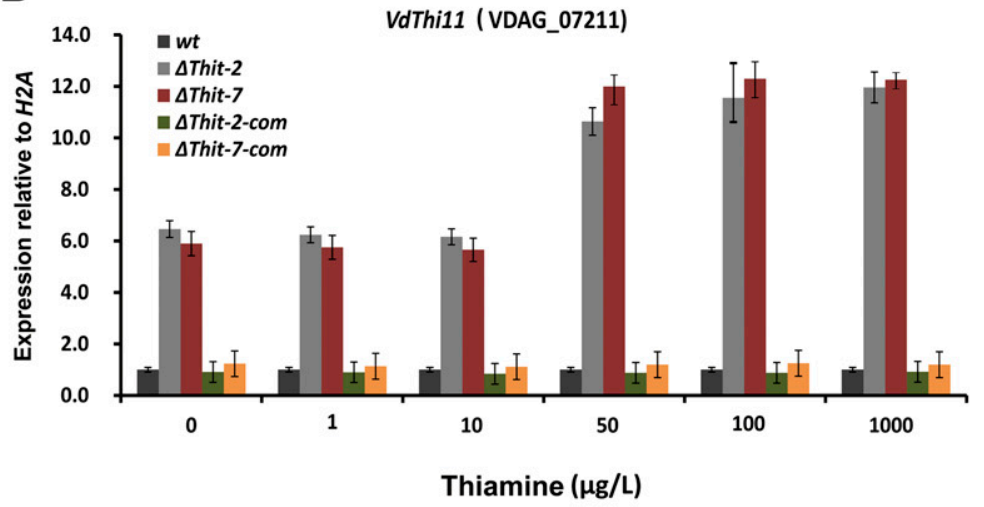

Fig. 9. Expression of de novo thiamine synthesis genes (VdSti35 [VDAG_01137] and Vdthil1 [VDAG_07211]) in the Vd $\Delta$ Thit (Vd $\Delta$ Thit-2 and Vd $\Delta$-7) mutant

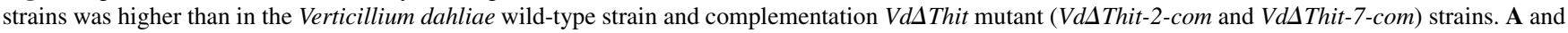
B, Quantitative real-time reverse-transcription polymerase chain reaction was used to measure VdSti35 and $\mathbf{C}$ and $\mathbf{D}$, Vdthill expression levels. The constitutively expressed $\beta$-tubulin gene was used as an internal standard. Total RNA was extracted from the mycelia of 7-day-old $V$. dahliae wild-type strain,

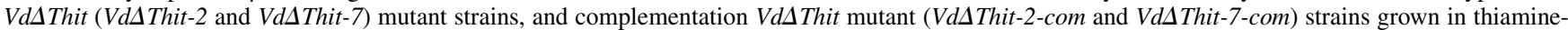
free (A and $\mathrm{C}$ ) and thiamine-supplemented (B and D; 1 to 1,000 $\mu \mathrm{g}$ per liter) liquid complete medium. Values are means \pm standard deviation of three independent experiments performed in duplicate. Asterisks (**) indicate significant difference in Student's $t$ test at $P<0.01$. 
2006). Our HPLC-MS and GC-MS results demonstrated that the pyruvate metabolism intermediates of the $V d \Delta$ Thit mutant strains, acetoin and acetyl-CoA, were markedly reduced, affecting the metabolism of intermediates in the Calvin cycle, and may be the cause of the sensitivity to stress and of decreased virulence of $V d \Delta$ Thit mutant strains. Furthermore, acetoin and acetyl-CoA in the VdsThit mutant strains were not detectable by HPLC-MS or GC-MS. We speculate that acetoin and acetyl-CoA may be related to the regulation of pyruvate metabolism, but further study is needed to explore how they contribute to regulating pyruvate metabolism in $V$. dahliae. The HPLC-MS and GC-MS results further confirmed that uptake of exogenous thiamine by the thiamine transporter protein is the main way that $V$. dahliae obtains thiamine; the thiamine de novo synthesis pathway is only an accessory pathway in $V$. dahlia.

Verticillium, a vascular pathogen, inhabits the vascular tissue of a host plant during colonization and infection because the xylem sap is the only source of accessible nutrients, including water, inorganic compounds, amino acids, and organic acids (Floerl et al. 2012; Iven et al. 2012; Kehr et al. 2005; Ratzinger et al. 2009; Satoh et al. 1998; Singh et al. 2010, 2012). Fungi acquire nutrients from their environment by their uptake systems (Ratzinger et al. 2009; Timpner et al. 2013), which include numerous ion channel systems in the plasma membrane for the uptake and transport of nutrient elements. In Verticillium longisporum, to absorb and use the amino acids present in the plant xylem tissue, cross-pathway control is required for acquiring amino acids from the imbalanced supply in the xylem so that it can further colonize the host (Singh et al. 2010; Timpner et al. 2013). In this study, we described the first construction of a $V$. dahliae mutant strain for the plasma-membrane protein transporting extracellular thiamine into the cell. The GFP-tagged strain $V d \Delta T h i t-G F P$ was deficient in early infection processes and few strains could successfully colonize and penetrate the root epidermis of $N$. benthamiana. Furthermore, at later stages of infection, only a few hyphae and conidia were observed in the xylem vessels of the roots. Growth of the GFP-tagged strain $V d \Delta T h i t-G F P$ was restricted enough within the vascular system to impair the ability of the fungus to colonize the vascular system. These results indicate that VdThit in $V$. dahliae plays an important role in the penetration, infection, and colonization of its host. In short, deletion of Thit in $V$. dahliae markedly decreased virulence of the fungus in $N$. benthamiana.

The adaptation of $V$. dahliae to life inside vascular tissues of its plant hosts requires a large number of channel proteins to control the absorption of nutrients across the plasma membrane. In this study, the thiamine transporter protein was functionally characterized and, for the first time, the function of this protein and its contribution to pathogenicity of Verticillium spp. were evaluated, providing novel insights into the molecular pathogenic mechanisms of $V$. dahliae.

\section{MATERIALS AND METHODS}

Fungal strains, plant material, plasmids, and media.

$V$. dahliae 991 was provided by G. Jian from the Institute of Plant Protection, Chinese Academy of Agricultural Sciences in Beijing, China. Nicotiana benthamiana used in this study was preserved in our laboratory. V. dahliae 991 and its variants were cultivated on potato dextrose agar, CM (6 g of yeast extract, $6 \mathrm{~g}$ of casein acid hydrolysate, and $10 \mathrm{~g}$ of sucrose per liter), or Czapek-Dox agar (per liter, $3.0 \mathrm{~g}$ of $\mathrm{NaNO}_{3}, 0.5 \mathrm{~g}$ of $\mathrm{MgSO}_{4} \cdot 7 \mathrm{H}_{2} \mathrm{O}$, $0.5 \mathrm{~g}$ of KCl, $0.01 \mathrm{~g}$ of $\mathrm{FeSO}_{4} \cdot 7 \mathrm{H}_{2} \mathrm{O}, 1.0 \mathrm{~g}$ of $\mathrm{K}_{2} \mathrm{HPO}_{4}$, and $15 \mathrm{~g}$ of agar). $N$. benthamiana plants were grown in a growth chamber at 23 to $25^{\circ} \mathrm{C}$, with $16 \mathrm{~h}$ of light and $8 \mathrm{~h}$ of dark, and 60 to $70 \%$ relative humidity. Agrobacterium tumefaciens AGL1 and plasmids pGKO2, pCAM-neo, pCM-Hyg, and pCAMgfp were provided by X. Dai from the Institute of Agro-products Processing Science
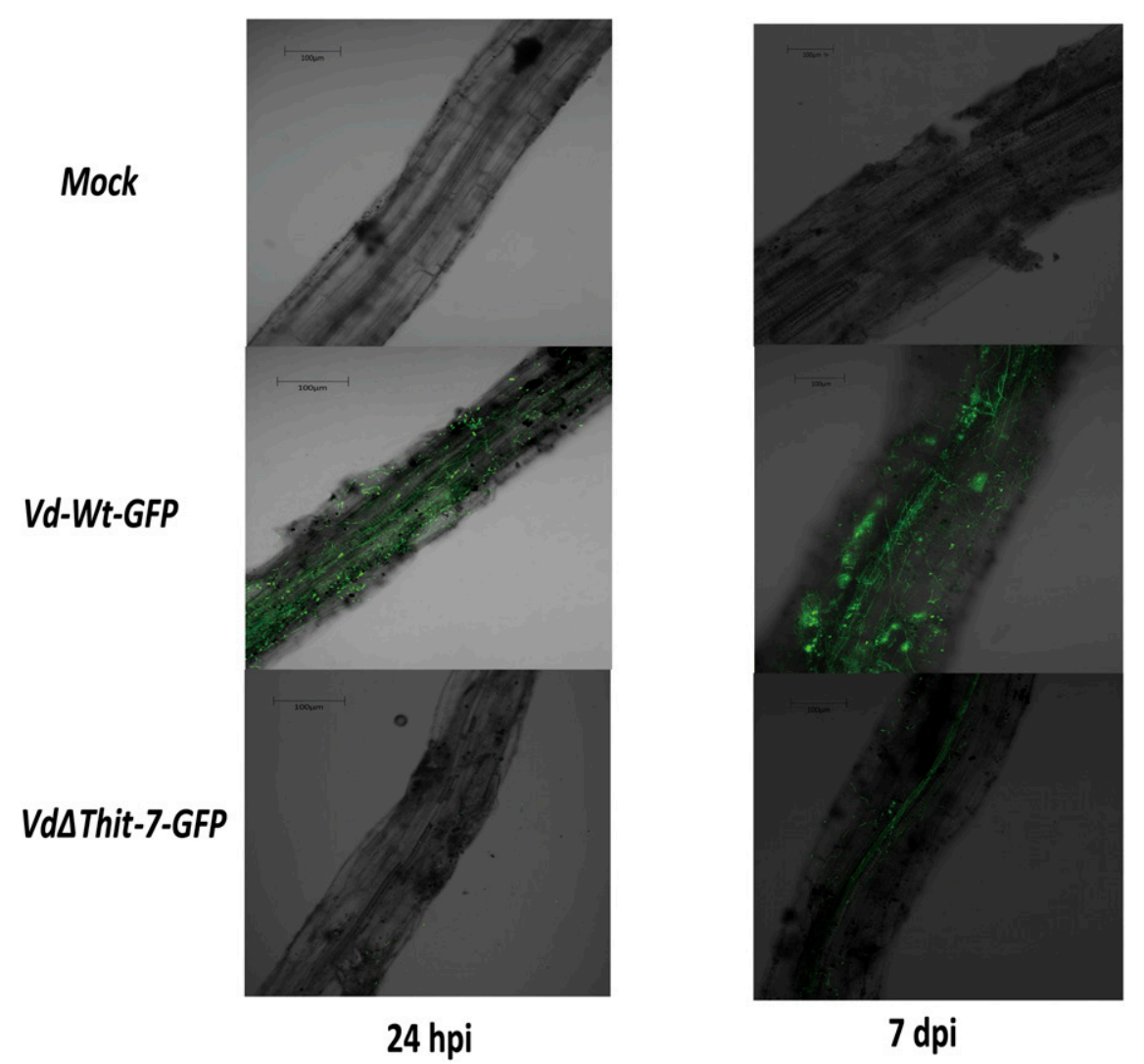

Fig. 10. Infection and fungal colonization of roots by the VdAThit-7-GFP mutant strains and $V d$-wt-GFP strain, $24 \mathrm{~h}$ (left) or 7 days (right) after inoculation. 
and Technology, Chinese Academy of Agricultural Sciences in Beijing, China.

\section{Plasmid construction and fungal transformation.}

The VdThit knockout cassette was constructed using InFusion cloning as described previously (Frandsen et al. 2012; Qi et al. 2015). Briefly, the 2.6-kb neo cassette was amplified from pCAM-neo using primer pair neo-F and neo-R (Table 1). A 1,071-bp 5' flanking region and a 1,012-bp 3' flanking region of VdThit were amplified from genomic DNA with primer pairs VdThit-5F and VdThit-5R and VdThit-3F and VdThit-3R, respectively (Table 1). Vector pGKO2 (Khang et al. 2005), carrying the HSVtk (herpes simplex virus thymidine kinase) gene as a negative selection marker against ectopic transformants, was digested with EcoRI or HindIII. For introducing compatible ends for cloning, primer pair VdThit-5F and VdThit-3R contained 15-bp-long $5^{\prime}$ overhangs identical to the corresponding pGKO2 sequence digested with EcoRI or HindIII. Similarly, primer pair VdThit-5R and VdThit-3F contained 15 -bp 5' overhangs identical to those in the neo-F and neo- $R$ sequences. Next, all three amplicons (the 5' flanking region, the neo cassette, and the $3^{\prime}$ flanking region) and the digested pGKO2 were fused together with an in-fusion enzyme (Clontech, Mountain View, CA, U.S.A.) to create the pGn-VdThit plasmid (pGKO2 [EcoRI]::VdThit-5'::neo::VdThit-3'::pGKO2 [HindIII] was fused according to the instructions for the In-Fusion drydown PCR cloning kit [Clontech]; each gene fragment contained 15-bp 5' overhangs [Fig. 1 A]). Similarly, for generating a VdThit complementation vector, the TrpC-promoter sequence (amplified using the primers C-TrpC-F and C-TrpC-R, [Table 1]), the full-length VdThit cDNA sequence (amplified with primers C-VdThit-F and C-VdThit-R [Table1]), the Nos-terminator fragment (with primers C-Nos-F and C-Nos-R [Table 1]), and the digested pCM-Hyg (carrying the hygromycin $\mathrm{B}$ resistance gene $[h p h]$ cassette, digested with $K p n \mathrm{I}$ and $X b a \mathrm{I}$ ) were fused together with an In-Fusion enzyme to construct the pCM-HygVdThit plasmid.

\section{Agrobacterium tumefaciens-mediated transformation (ATMT).}

The plasmids pGn-VdThit (carrying the neomycin-resistance gene) and pCM-Hyg-VdThit were introduced into Agrobacterium tumefaciens AGL1 via electroporation. ATMT of $V$. dahliae Vd991 with pGn-VdThit and the VdDThit mutant strains with pCM-Hyg-VdThit was done as described previously (Hooykaas et al. 1979; Mullins et al. 2001). Similarly, plasmid pCAMgfp (with the eGFP gene and $h p h$ ) was introduced into the wild-type strain and the VdsThit mutant strains via ATMT, to observe the infection process in plants.

\section{Confirmation of VdThit gene disruption or complementation.}

Transformants were initially screened for neomycin phosphotransferase resistance to obtain the desired mutant strain, using PCR with primers VdThit-J-F and VdThit-J-R (Table 1). Replacement of VdThit with hygromycin B resistance was further analyzed by PCR, using primers neo-J-F and neo-J-R (Table 1). The selected complementation transformants were PCR-amplified, using primers VdThit-J-F and VdThit-J-R (Table 1).

\section{Growth, conidiogenesis, and germination assays.}

$V d \Delta$ Thit mutant strains were characterized by measuring colony diameter every 2 to 3 days and recording colony morphology on minimal medium (MM) (per liter, $6 \mathrm{~g}$ of $\mathrm{NaNO}_{3}, 0.52 \mathrm{~g}$ of $\mathrm{KCl}$, $0.152 \mathrm{~g}$ of $\mathrm{MgSO}_{4}, 1.52 \mathrm{~g}$ of $\mathrm{KH}_{2} \mathrm{PO}_{4}, 0.01 \mathrm{~g}$ of thiamine, $10 \mathrm{ml}$ of trace elements, and $15 \mathrm{~g}$ of agar) amended with different carbon sources (per liter, $10 \mathrm{~g}$ of xylose, galactose, pectin, or starch or
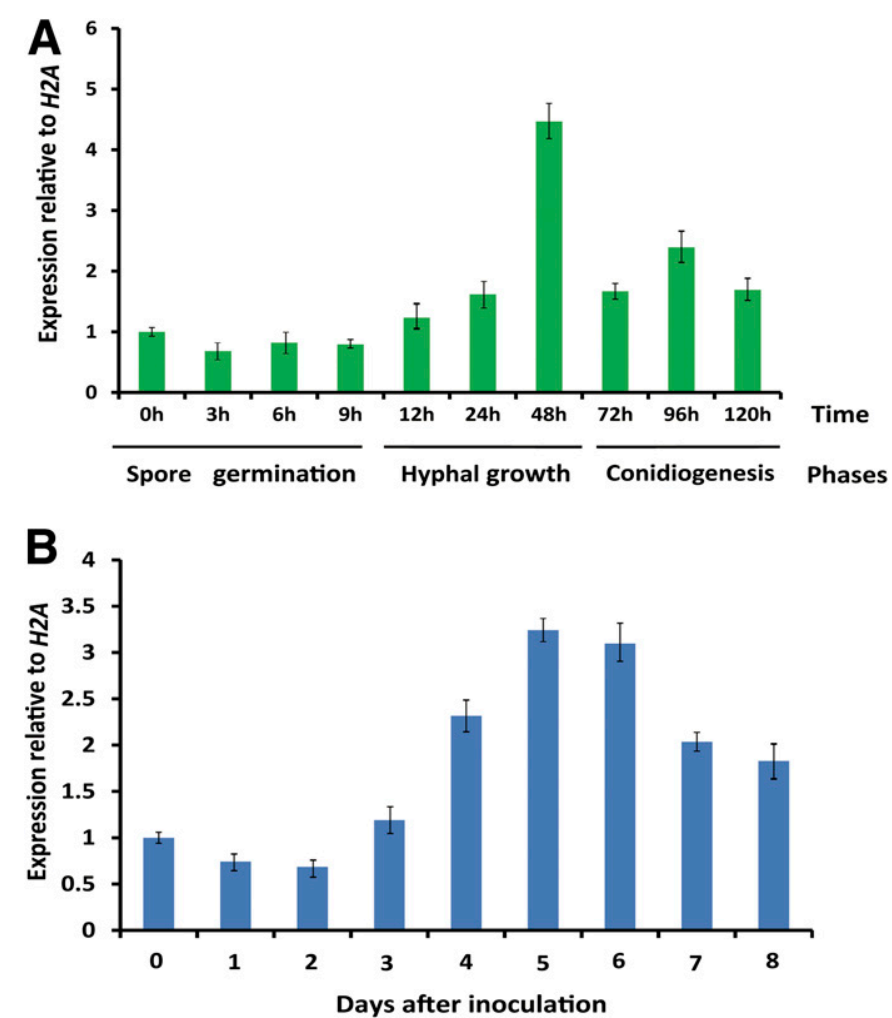

Fig. 11. Expression pattern analysis of VdThit gene during the development of Verticillium dahliae on media and assays of plant infection. A, Analysis of VdThit gene expression during conidial germination (at $0,3,6$, and $9 \mathrm{~h}$ ), hyphal growth (at 12, 24, and $48 \mathrm{~h}$ ), and conidiogenesis (at 72, 96, and 120 h) in liquid complete medium. B, Total RNA was extracted individually from hypocotyls of plants of Nicotiana benthamiana at 1, 2, 3, 4, 5, 6, 7, 8, and 9 days infected with $V$. dahliae to estimate the VdThit gene expression by quantitative reverse transcription-polymerase chain reaction. 
$30 \mathrm{~g}$ of sucrose [Puhalla and Mayfield 1974; Tzima et al. 2011]). Conidial production by wild-type strain 991 of $V$. dahliae, comple-

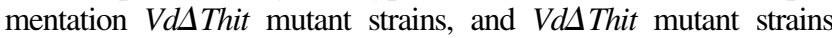
cultured on Czapek-Dox agar were assessed as described previously (Qi et al. 2015). For germination tests, $10^{4}$ spores per milliliter of the $V$. dahliae wild-type strain, complementation VdsThit mutant strains, and VdsThit mutant strains were added to liquid $\mathrm{CM}$ and, then, were shaken for $30 \mathrm{~h}$ at $140 \mathrm{rpm}$ at $25^{\circ} \mathrm{C}$. At 5 -h intervals, 100 conidia were examined for germination, and percent germination was calculated (Tzima et al. 2012). Each assay was done three times.

\section{Phenotypic analysis under stress conditions.}

Fresh conidia, 1,000 each from the $V$. dahliae wild-type

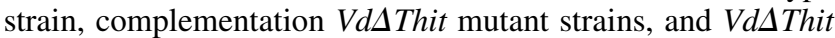
mutant strains were plated onto Czapek-Dox agar plates and were incubated at $25^{\circ} \mathrm{C}$ for $1 \mathrm{~h}$. For determining conidial survival of these strains in response to $\mathrm{UV}$, conidia were exposed to $\mathrm{UV}$ radiation at $50,100,150$, and $200 \mathrm{~J} \mathrm{~m}^{-2}$, using a UV Crosslinker (Spectrolinker XL-1000A, Spectronics Corp., Westbury, NY, U.S.A.), and then, were incubated for 3 days at $25^{\circ} \mathrm{C}$ before CFU were counted on each plate. The UV-irradiation assay was repeated three times independently.

Next, $2 \mu \mathrm{l}$ of conidial suspensions $\left(5 \times 10^{6}\right.$ spores per milliliter) of the $V$. dahliae wild-type strain, complementation $V d \Delta T$ Thit mutant strains, and VdsThit mutant strains were dropped onto the center of Czapek-Dox agar plates supplemented with $10 \mu \mathrm{g}$ of menadione per liter (oxidative stress) or $1 \mathrm{M}$ sorbitol (osmotic stress) or without menadione or sorbitol. The plates were incubated at $25^{\circ} \mathrm{C}$ for 7 days, and colony phenotypes were then observed. The assay was repeated three times independently.

\section{qRT-PCR reaction.}

Transcript levels of the genes VdSti35 (VDAG_01137) and VdThi11 (VDAG_07211) for de novo thiamine synthesis were examined in the $\bar{V} d \Delta$ Thit mutant strains, wild-type strain, and complementation $V d \Delta$ Thit mutant strains grown in liquid CM. Total RNA was extracted from cells, using an RNA miniprep kit (Axygen, Union City, CA, U.S.A.) according to the manufacturer's instructions. cDNA was synthesized from purified RNA using a Toyobo RT kit (Osaka, Japan). The qRT-PCR reaction was performed with the SYBR Fast qPCR kit (Kapa Biosystems, Boston). Transcript levels of VdSti35 and VdThill were quantified relative to the constitutively expressed $\beta$-tubulin gene (DQ266153), which was amplified with primers VdBt-F and VdBt-dn, using qRTPCR in an ABI7500 PCR thermocycler (Applied Biosystems, Foster City, CA, U.S.A.). The qRT-PCR analysis of the VdsThit mutant strains and wild-type strain was done three times.

RNA was extracted from conidia or hyphae of $V$. dahliae harvested after $0,3,6,9,12,24,72,96$, and $120 \mathrm{~h}$ of growth in liquid CM, for identifying distinct stages of fungal development, and VdThit gene expression was analyzed by qRT-PCR. Total RNA was extracted from individual hypocotyls of diseased plants daily from day 1 to day 9 after inoculation to estimate VdThit gene expression, by qRT-PCR, using the SYBR Fast qPCR kit. The qRT-PCR analysis of VdThit gene expression in the wild-type strain was done three times.

\section{Infection assays and quantification \\ of $V d \Delta T h i t$ fungal biomass in plant tissue, using qRT-PCR.}

Infection assays and quantification of the VdsThit fungal biomass in plant tissue, using qRT-PCR, were performed as described previously (Qi et al. 2015). N. benthamiana seedlings with 6 to 7 true leaves were removed from the soil and roots were dipped into a conidial suspension $\left(5 \times 10^{6}\right.$ spores per milliliter $)$ of $V$. dahliae wild-type strain, complementation VdsThit mutant strains, or $V d \Delta T h i t$ mutant strains (Qi et al. 2015). Disease level was recorded and calculated at 8,10 , and 12 dpi. Total DNA was extracted individually from the hypocotyls of diseased plants at 12 days after inoculation, to estimate fungal biomass by qRTPCR, using the SYBR Fast qPCR kit (Kapa Biosystems). To normalize any differences in DNA template amounts, the $N$. benthamiana actin gene was amplified with primer pair $\mathrm{Nb}$ actin-F and Nb-actin-R (Table 1) and was used as an internal standard. Primer pair Vd-F and Vd-R (Table 1), designed based on the ITS1 and ITS2 regions of the ribosomal RNA genes (Z29511) of $V$. dahliae, were used to quantify fungal DNA in each sample. Fungal outgrowth of the infected plant material was measured from harvested stems as described previously (Santhanam and Thomma 2013). The infection assay and qRTPCR analysis of $N$. benthamiana tissue were done three times (with 10 plants per strain).

\section{HPLC-MS and GC-MS analysis of the $V d \Delta$ Thit mutant strains.}

For chemical assays of pyruvate metabolism intermediates in cell extracts, mycelial samples grown for 6 days in liquid CM were harvested and were powdered in liquid nitrogen. A highly sensitive HPLC-MS was performed, as described previously, to analyze acetyl-CoA in the Krebs cycle of VdDThit mutant and wild-type strains (Hayashi et al. 2006). A previously published GC-MS method (Güler-Akin et al. 2009), with slight modifications, was used to analyze acetoin in pyruvate metabolism in the VdSThit mutant strain and wild-type strain. The extracts from $V$. dahliae wild-type strain or VdDThit mutant strain were analyzed using a Shimadzu GCMS-QP2010 Plus system (Shimadzu, Sao Paulo, Brazil) and a Chromolith Performance DB-WAX column $(30 \mathrm{~m} \times 0.25 \mathrm{~mm} \times 0.25 \mu \mathrm{m})$ and hydrogen carrier gas (flow rate $1 \mathrm{ml} \mathrm{min}^{-1}$ ) with injector temperature of $250^{\circ} \mathrm{C}$ and ion-source temperature of $200^{\circ} \mathrm{C}$. The oven temperature was programmed from $40^{\circ} \mathrm{C}$ (isothermal for $2 \mathrm{~min}$ ), with an increase of $5^{\circ} \mathrm{C} \mathrm{min}{ }^{-1}$ to $120^{\circ} \mathrm{C}$, and then, $10^{\circ} \mathrm{C} \mathrm{min}{ }^{-1}$ to $200^{\circ} \mathrm{C}$, ending with a 5 -min isothermal at $200^{\circ} \mathrm{C}$. Ions were detected in full-scan monitoring mode from 35 to $500 \mathrm{~m} / \mathrm{z}$. The

Table 1. Primers used in this study

\begin{tabular}{ll}
$\begin{array}{l}\text { Primer } \\
\text { name }\end{array}$ & \multicolumn{1}{c}{ Sequences $\left(\mathbf{5}^{\prime} \rightarrow \mathbf{3}^{\prime}\right)$} \\
\hline Thit-5F & $\begin{array}{l}\text { GTACCCAATTCGAATTCTTAGTGCCTGGACGTT } \\
\text { TTAGTGA }\end{array}$ \\
Thit-5R & CAAGACAGCCCGCAAACCCGGGGTGGTGAGGATG \\
Thit-3F & CCCAGAATGCACAGGTACCTTCCCCGACGTG \\
& TTTTCA \\
Thit-3R & GACGGTATCGATAAGCTTTAGCCGGACGCGTTG \\
& TAGGT \\
C-TrpC-F & TTGAAGGAGCATTTTTGGGC \\
C-TrpC-R & ATCGATGCTTGGGTAGAATAGGT \\
C-VdThit-F & ATGTGGAAGAACATTGTCGAGAAG \\
C-VdThit-R & TCACTCCCTCAAGTACCGTATCTTC \\
C-Nos-F & AGATGCCGACCGGGATCCACTT \\
C-Nos-R & TTATCTTTGCGACCCAGGG \\
neo-F & GTTTGCGGGCTGTCTTGACG \\
neo-R & TACCTGTGCATTCTGGGTAA \\
Thit-J-F & GCTATGATCAGCAGCTGGAGCTC \\
Thit-J-R & ACCCCCTTGTTCCTCGCCTGAT \\
noe-J-F & GCGGTTCAGAAGCACCTCGA \\
noe-J-R & TATCTTTGCGAACCCAGG \\
VdBt-F & TTCCCCCGTCTCCACTTCTTCATG \\
VdBt-R & GACGAGATCGT TCATGTTGAACTC \\
Vdsti35-F & GGCCCCCAAGACCAACAGC \\
Vdsit35-R & GAGCGAGTAGGCGGCGGAGAG \\
Vdthi11-F & TCATGGGCTCGGACCTCAACC \\
Vdthi11-R & GACGGCAGCGATCTCAATGG \\
Nb-actin-F & GGACCTTTATGGAAACATTGTGCTCAGT \\
Nb-actin-R & CCAAGATAGAACCTCCAATCCAGACAC \\
Vd- F & CCGCCGGTCCATCAGTCTCTCTGTTTATAC \\
Vd- R & CGCCTGCGGGACTCCGATGCGAGCTGTAAC \\
\hline &
\end{tabular}


mass spectra of the products detected by GC-MS were then compared with those in the National Institute of Standards and Technology library (NIST011). Quantities were calculated using an external standard and area normalization method.

\section{Growth, conidiation, and virulence of the VdDThit mutant strains under different exogenous thiamine levels.}

The VdsThit mutant strains were characterized according to their colony diameter, conidia production, and virulence (infection assays) on Czapek-Dox agar plates supplemented with 1 to $1,000 \mu \mathrm{g}$ of exogenous thiamine per liter or without thiamine, as described above. The experiment was repeated three times.

\section{Microscopic observation of initial infection.}

Roots of $30 \mathrm{~N}$. benthamiana seedlings with three to four true leaves were inoculated with $V d$-wt-GFP strain and $V d \Delta T h i-7-G F P$ mutant strain as described earlier, respectively. At $24 \mathrm{hpi}$ and $7 \mathrm{dpi}$, $N$. benthamiana plants were uprooted and were gently washed in tap water. The roots of the $N$. benthamiana plants were cut vertically and were microscopically examined using LSCM (LSM 700; Zeiss, Jena, Germany). The experiment was done three times.

\section{ACKNOWLEDGMENT}

This work was supported by a grant from the Key Project for Breeding Genetic Modified Organisms (2012ZX08009-001).

\section{LITERATURE CITED}

Ahn, I. P., Kim, S., and Lee, Y. H. 2005. Vitamin B1 functions as an activator of plant disease resistance. Plant Physiol. 138:1505-1515.

Butterworth, R. F. 2003. Thiamin deficiency and brain disorders. Nutr. Res. Rev. 16:277-284.

Cai, Y. F., He, X. H., Mo, J. C., Sun, Q., Yang, J. P., and Liu, J. G. 2009. Molecular research and genetic engineering of resistance to Verticillium wilt in cotton: A review. Afr. J. Biotechnol. 8:7363-7372.

Callejas-Negrete, O. A., Torres-Guzmán, J. C., Padilla-Guerrero, I. E., EsquivelNaranjo, U., Padilla-Ballesteros, M. F., García-Tapia, A., Schrank, A., Salazar-Solís, E., Gutiérrez-Corona, F., and González-Hernández, G. A. 2015. The Adhl gene of the fungus Metarhizium anisopliae is expressed during insect colonization and required for full virulence. Microbiol. Res. 172:57-67.

Chu, J., Li, W.-F., Cheng, W., Lu, M., Zhou, K.-H., Zhu, H.-Q., Li, F.-G. and Zhou, C.-Z. 2015. Comparative analyses of secreted proteins from the phytopathogenic fungus Verticillium dahliae in response to nitrogen starvation. Biochim. Biophys. Acta 1854:437-448.

Du, Y., Hong, L., Tang, W., Li, L., Wang, X., Ma, H., Wang, Z., Zhang, H., Zheng, X., and Zhang, Z. 2014. Threonine deaminase Mollv1 is important for conidiogenesis and pathogenesis in the rice blast fungus Magnaporthe oryzae. Fungal Genet. Biol. 73:53-60.

Dutta, B., Huang, W., Molero, M., Kekuda, R., Leibach, F. H., Devoe, L. D. Ganapathy, V., and Prasad, P. D. 1999. Cloning of the human thiamine transporter, a member of the folate transporter family. J. Biol. Chem. 274:31925-31929.

Enjo, F., Nosaka, K., Ogata, M., Iwashima, A., and Nishimura, H. 1997. Isolation and characterization of a thiamin transport gene, THI1O, from Saccharomyces cerevisiae. J. Biol. Chem. 272:19165-19170.

Eynck, C., Koopmann, B., Grunewaldt-Stoecker, G., Karlovsky, P., and Von Tiedemann, A. 2007. Differential interactions of Verticillium longisporum and V. dahliae with Brassica napus detected with molecular and histological techniques. Eur. J. Plant Pathol. 118:259-274.

Floerl, S., Majcherczyk, A., Possienke, M., Feussner, K., Tappe, H., Gatz, C., Feussner, I., Kües, U., and Polle, A. 2012. Verticillium longisporum infection affects the leaf apoplastic proteome, metabolome, and cell wall properties in Arabidopsis thaliana. PLoS One 7:e31435.

Fradin, E. F., and Thomma, B. P. 2006. Physiology and molecular aspects of Verticillium wilt diseases caused by V. dahliae and V. albo-atrum. Mol. Plant Pathol. 7:71-86.

Frandsen, R. J., Frandsen, M., and Giese, H. 2012. Targeted gene replacement in fungal pathogens via Agrobacterium tumefaciens-mediated transformation. Pages 17-45 in: Plant Fungal Pathogens: Methods and Protocols. M. D. Bolton and B. P. H. J. Thomma, eds. Humana Press, New York.

Friedrich, W. 1987. Thiamin (Vitamin B1, aneurin). Pages 240-258 in: Handbuch der Vitamine. Urban and Schwartzenberg, Munich.
Gomes, D. S., Lopes, M. A., Menezes, S. P., Ribeiro, L. F., Dias, C. V. Andrade, B. S., de Jesus, R. M., Pires, A. B., Goes-Neto, A., and Micheli, F. 2016. Mycelial development preceding basidioma formation in Moniliophthora perniciosa is associated to chitin, sugar and nutrient metabolism alterations involving autophagy. Fungal Genet. Biol. 86 33-46.

Goyer, A. 2010. Thiamine in plants: Aspects of its metabolism and functions. Phytochemistry 71:1615-1624.

Güler-Akin, M. B., Serdar Akin, M., and Korkmaz, A. 2009. Influence of different exopolysaccharide-producing strains on the physicochemical, sensory and syneresis characteristics of reduced-fat stirred yoghurt. Int. J. Dairy Technol. 62:422-430.

Harris, L. J., Balcerzak, M., Johnston, A., Schneiderman, D., and Ouellet, T. 2016. Host-preferential Fusarium graminearum gene expression during infection of wheat, barley, and maize. Fungal Biol. 120:111-123.

Hayashi, M., Mizoguchi, H., Ohnishi, J., Mitsuhashi, S., Yonetani, Y., Hashimoto, S. I., and Ikeda, M. 2006. A leuC mutation leading to increased L-lysine production and rel-independent global expression changes in Corynebacterium glutamicum. Appl. Microbiol. Biot. 72 783-789.

Hillocks, R. J. 1992. Cotton Diseases. CAB International, Wallingford, U.K. Hogenhout, S. A., Van der Hoorn, R. A., Terauchi, R., and Kamoun, S. 2009. Emerging concepts in effector biology of plant-associated organisms. Mol. Plant-Microbe Interact 22:115-122.

Hohmann, S., and Meacock, P. A. 1998. Thiamin metabolism and thiamin diphosphate-dependent enzymes in the yeast Saccharomyces cerevisiae: Genetic regulation. BBA-Protein Struct. M. 1385:201-219.

Hooykaas, P. J., Roobol, C., and Schilperoort, R. A. 1979. Regulation of the transfer of Ti plasmids of Agrobacterium tumefaciens. Microbiology 110:99-109.

Hoppenau, C. E., Tran, V. T., Kusch, H., Aßhauer, K. P., Landesfeind, M. Meinicke, P., Popovac, B., Braus-Stromeyer, S. A., and Braus, G. H 2014. Verticillium dahliae VdTHI4, involved in thiazole biosynthesis, stress response and DNA repair functions, is required for vascular disease induction in tobacco. Environ. Exp. Bot. 108:14-22.

Ikegami, A., Nakasone, K., Kato, C., Nakamura, Y., Yoshikawa, I., Usami, R., and Horikoshi, K. 2000. Glutamine synthetase gene expression at elevated hydrostatic pressure in a deep-sea piezophilic Shewanella violacea. FEMS (Fed. Eur. Microbiol. Soc.) Microbial. Lett. 192:91-95.

Iven, T., König, S., Singh, S., Braus-Stromeyer, S. A., Bischoff, M., Tietze, L. F., Braus, G. H., Lipka, V., Feussner, I., and Dröge-Laser, W. 2012. Transcriptional activation and production of tryptophan-derived secondary metabolites in arabidopsis roots contributes to the defense against the fungal vascular pathogen Verticillium longisporum. Mol. Plant 5:1389-1402.

Iwashima, A., Nishimura, H., and Nose, Y. 1979. Soluble and membranebound thiamine-binding proteins from Saccharomyces cerevisiae. Biochim. Biophys. Acta 557:460-468.

Kehr, J., Buhtz, A., and Giavalisco, P. 2005. Analysis of xylem sap proteins from Brassica napus. BMC Plant Biol. 5:11.

Khang, C. H., Park, S. Y., Lee, Y. H., and Kang, S. 2005. A dual selection based, targeted gene replacement tool for Magnaporthe grisea and Fusarium oxysporum. Fungal Genet. Biol. 42:483-492.

Klosterman, S. J., Atallah, Z. K., Vallad, G. E., and Subbarao, K. V. 2009. Diversity, pathogenicity, and management of verticillium species. Annu. Rev. Phytopathol. 47:39-62.

Klosterman, S. J., Subbarao, K. V., Kang, S., Veronese, P., Gold, S. E., Thomma, B. P., Chen, Z., Henrissat, B., Lee, Y. H., Park, J., GarciaPedrajas, M. D., Barbara, D. J., Anchieta, A., de Jonge, R., Santhanam, P., Maruthachalam, K., Atallah, Z., Amyotte, S. G., Paz, Z., Inderbitzin, P., Hayes, R. J., Heiman, D. I., Young, S., Zeng, Q., Engels, R., Galagan, J., Cuomo, C. A., Dobinson, K. F., and Ma, L. J. 2011. Comparative genomics yields insights into niche adaptation of plant vascular wilt pathogens. PLoS Pathog. 7:e1002137.

Kong, L. A., Yang, J., Li, G. T., Qi, L. L., Zhang, Y. J., Wang, C. F., Zhao, W. S., Xu, J. R., and Peng, Y. L. 2012. Different chitin synthase genes are required for various developmental and plant infection processes in the rice blast fungus Magnaporthe oryzae. PLoS Pathog. 8:e1002526.

Lagarde, W. H., Underwood, L. E., Moats-Staats, B. M., and Calikoglu, A. S. 2004. Novel mutation in the SLC19A2 gene in an AfricanAmerican female with thiamine-responsive megaloblastic anemia syndrome. Am. J. Med. Genet. A. 125A:299-305.

Larkin, J. R., Zhang, F., Godfrey, L., Molostvov, G., Zehnder, D., Rabbani, N., and Thornalley, P. J. 2012. Glucose-induced down regulation of thiamine transporters in the kidney proximal tubular epithelium produces thiamine insufficiency in diabetes. PLoS One 7:e53175.

Lee, J., Leslie, J. F., and Bowden, R. L. 2008. Expression and function of sex pheromones and receptors in the homothallic ascomycete Gibberella zeae. Eukaryot. Cell 7:1211-1221. 
Lu, G., Dobritzsch, D., Baumann, S., Schneider, G., and König, S. 2000. The structural basis of substrate activation in yeast pyruvate decarboxylase. A crystallographic and kinetic study. Eur. J. Biochem. 267:861-868.

Luo, X., Xie, C., Dong, J., Yang, X., and Sui, A. 2014. Interactions between Verticillium dahliae and its host: Vegetative growth, pathogenicity, plant immunity. Appl. Microbiol. Biotechnol. 98:6921-6932.

Moe, O. A., Jr., Lerner, D. A., and Hammes, G. G. 1974. Fluorescence energy transfer between the thiamine diphosphate and flavine adenine dinucleotide binding sites on the pyruvate dehydrogenase multienzyme complex. Biochemistry 13:2552-2557.

Mojzita, D., and Hohmann, S. 2006. Pdc2 coordinates expression of the THI regulon in the yeast Saccharomyces cerevisiae. Mol. Genet. Genomics 276:147-161.

Mullins, E. D., Chen, X., Romaine, P., Raina, R., Geiser, D. M., and Kang, S. 2001. Agrobacterium-mediated transformation of Fusarium oxysporum: An efficient tool for insertional mutagenesis and gene transfer. Phytopathology 91:173-180.

Oishi, K., Hofmann, S., Diaz, G. A., Brown, T., Manwani, D., Ng, L., Young, R., Vlassara, H., Ioannou, Y. A., Forrest, D., and Gelb, B. D. 2002. Targeted disruption of Slc19a2, the gene encoding the high-affinity thiamin transporter Thtr-1, causes diabetes mellitus, sensorineural deafness and megaloblastosis in mice. Hum. Mol. Genet. 11:2951-2960.

Pegg, G. F., and Brady, B. L., eds. 2002. Verticillium Wilts. CABI Publishing, Wallingford, U.K.

Puhalla, J. E., and Mayfield, J. E. 1974. The mechanism of heterokaryotic growth in Verticillium dahliae. Genetics 76:411-422.

Qi, X., Su, X., Guo, H., Qi, J., and Cheng, H. 2015. A ku70 null mutant improves gene targeting frequency in the fungal pathogen Verticillium dahliae. World J. Microbiol. Biotechnol. 31:1889-1897.

Rapala-Kozik, M. 2011. Vitamine B1 (thiamine): A cofactor for enzymes involved in the main metabolic pathways and an environmental stress protectant. Adv. Bot. Res. 58:37-91.

Rapala-Kozik, M., Kowalska, E., and Ostrowska, K. 2008. Modulation of thiamine metabolism in Zea mays seedlings under conditions of abiotic stress. J. Exp. Bot. 59:4133-4143.

Rapala-Kozik, M., Wolak, N., Kujda, M., and Banas, A. K. 2012. The upregulation of thiamine (vitamin B1) biosynthesis in Arabidopsis thaliana seedlings under salt and osmotic stress conditions is mediated by abscisic acid at the early stages of this stress response. BMC Plant Biol. 12:2.

Ratzinger, A., Riediger, N., von Tiedemann, A., and Karlovsky, P. 2009. Salicylic acid and salicylic acid glucoside in xylem sap of Brassica napus infected with Verticillium longisporum. J. Plant Res. 122:571-579.

Reusche, M., Thole, K., Janz, D., Truskina, J., Rindfleisch, S., Drübert, C., Polle, A., Lipka, V., and Teichmann, T. 2012. Verticillium infection triggers VASCULAR-RELATED NAC DOMAIN7-dependent de novo xylem formation and enhances drought tolerance in Arabidopsis. Plant Cell 24:3823-3837.

Rindi, G. 1992. Some aspects of thiamin transport in mammals. J. Nutr. Sci. Vitaminol. (Tokyo) 38:379-382.

Ruiz-Roldán, C., Puerto-Galán, L., Roa, J., Castro, A., Di Pietro, A., Roncero, M. I., and Hera, C. 2008. The Fusarium oxysporum sti35 gene functions in thiamine biosynthesis and oxidative stress response. Fungal Genet. Biol. 45:6-16.

Santhanam, P., and Thomma, B. P. H. J. 2013. Verticillium dahliae Sge1 differentially regulates expression of candidate effector genes. Mol. Plant-Microbe Interact 26:249-256.

Satoh, S., Kuroha, T., Wakahoi, T., and Inouye, Y. 1998. Inhibition of the formation of adventitious roots on cucumber hypocotyls by the fractions and methoxybenzyl glutamine from xylem sap of squash root. J. Plant Res. 111:541-546.
Schumacher, J. 2016. DHN melanin biosynthesis in the plant pathogenic fungus Botrytis cinerea is based on two developmentally regulated key enzyme (PKS)-encoding genes. Mol. Microbiol. 99:729-748.

Shao, W., Yang, Y., Zhang, Y., Lv, C., Ren, W., and Chen, C. 2015. Involvement of BcStr2 in methionine biosynthesis, vegetative differentiation, multiple stress tolerance and virulence in Botrytis cinerea. Mol. Plant Pathol. 17:438-447.

Singh, S., Braus-Stromeyer, S. A., Timpner, C., Tran, V. T., Lohaus, G., Reusche, M., Knüfer, J., Teichmann, T., von Tiedemann, A., and Braus, G. H. 2010. Silencing of Vlaro2 for chorismate synthase revealed that the phytopathogen Verticillium longisporum induces the crosspathway control in the xylem. Appl. Microbiol. Biotechnol. 85: 1961-1976.

Singh, S., Braus-Stromeyer, S. A., Timpner, C., Valerius, O., von Tiedemann, A., Karlovsky, P., Druebert, C., Polle, A., and Braus, G. H. 2012. The plant host Brassica napus induces in the pathogen Verticillium longisporum the expression of functional catalase peroxidase which is required for the late phase of disease. Mol. Plant-Microbe Interact 25: 569-581.

Singleton, C. K. 1997. Identification and characterization of the thiamine transporter gene of Saccharomyces cerevisiae. Gene 199:111-121.

Sriram, K., Manzanares, W., and Joseph, K. 2012. Thiamine in nutrition therapy. Nutr. Clin. Pract. 27:41-50.

Timpner, C., Braus-Stromeyer, S. A., Tran, V. T., and Braus, G. H. 2013. The $\mathrm{Cpc} 1$ regulator of the cross-pathway control of amino acid biosynthesis is required for pathogenicity of the vascular pathogen Verticillium longisporum. Mol. Plant-Microbe Interact 26:1312-1324.

Tzima, A. K., Paplomatas, E. J., Rauyaree, P., Ospina-Giraldo, M. D., and Kang, S. 2011. VdSNF1, the sucrose nonfermenting protein kinase gene of Verticillium dahliae, is required for virulence and expression of genes involved in cell-wall degradation. Mol. Plant-Microbe Interact 24:129-142.

Tzima, A. K., Paplomatas, E. J., Tsitsigiannis, D. I., and Kang, S. 2012. The $\mathrm{G}$ protein $\beta$ subunit controls virulence and multiple growth- and development-related traits in Verticillium dahliae. Fungal Genet. Biol. 49:271-283.

Yang, X., Ding, F., Zhang, L., Sheng, Y., Zheng, X., and Wang, Y. 2015. The importin $\alpha$ subunit PsIMPA1 mediates the oxidative stress response and is required for the pathogenicity of Phytophthora sojae. Fungal Genet. Biol. 82:108-115.

Ying, S. H., Ji, X. P., Wang, X. X., Feng, M. G., and Keyhani, N. O. 2014. The transcriptional co-activator multiprotein bridging factor 1 from the fungal insect pathogen, Beauveria bassiana, mediates regulation of hyphal morphogenesis, stress tolerance and virulence. Environ. Microbiol. 16:1879-1897.

Young, A. W., McWeeny, K. H., Hay, D. C., and Ellis, A. W. 1986. Matching familiar and unfamiliar faces on identity and expression. Psychol. Res. 48:63-68.

Zeier, J., Bernsdorff, F., Doering, A. C., Gruner, K., Bräutigam, A., and Schuck, S. 2015. Pipecolic acid orchestrates plant systemic acquired resistance and defense priming via salicylic acid-dependent and independent pathways. Plant Cell 28:201-129.

Zhao, P., Zhao, Y. L., Jin, Y., Zhang, T., and Guo, H. S. 2014. Colonization process of Arabidopsis thaliana roots by a green fluorescent proteintagged isolate of Verticillium dahliae. Protein Cell 5:94-98.

\section{AUTHOR-RECOMMENDED INTERNET RESOURCE}

Verticillium Comparative Genomics Project database: http://www.broadinstitute.org/annotation/genome/verticillium_dahliae 